\title{
MICROFLUIDIC DROPLET DETECTION VIA REGION-BASED AND SINGLE-PASS CONVOLUTIONAL NEURAL NETWORKS WITH COMPARISON TO CONVENTIONAL IMAGE ANALYSIS METHODOLOGIES
}

\section{A PREPRINT}

\author{
Gregory Rutkowski* \\ Ugelstad Laboratory \\ Department of Chemical Engineering \\ Norwegian University of Science and Technology \\ Trondheim, Norway \\ gregory.p.rutkowski@ntnu.no \\ Evan Unmann \\ Department of Computer Science \\ University of North Carolina at Charlotte \\ Charlotte, North Carolina, USA \\ eunmann@uncc.edu
}

\author{
Ilgar Azizov \\ Ugelstad Laboratory \\ Department of Chemical Engineering \\ Norwegian University of Science and Technology \\ Trondheim, Norway \\ ilgar.azizov@ntnu.no
}

\author{
Marcin Dudek \\ Ugelstad Laboratory \\ Department of Chemical Engineering \\ Norwegian University of Science and Technology \\ Trondheim, Norway \\ marcin.dudek@ntnu.no
}

\author{
Brian Arthur Grimes \\ Ugelstad Laboratory \\ Department of Chemical Engineering \\ Norwegian University of Science and Technology \\ Trondheim, Norway \\ brian.grimes@ntnu.no
}

June 1,2021

\begin{abstract}
As the complexity of microfluidic experiments and the associated image data volumes scale, traditional feature extraction approaches begin to struggle at both detection and analysis pipeline throughput. Deep-neural networks trained to detect certain objects are rapidly emerging as data gathering tools that can either match or outperform the analysis capabilities of the conventional methods used in microfluidic emulsion science. We demonstrate that various convolutional neural networks can be trained and used as droplet detectors in a wide variety of microfluidic systems. A generalized microfluidic droplet training and validation dataset was developed and used to tune two versions of the You Only Look Once (YOLOv3/YOLOv5) model as well as Faster R-CNN. Each model was used to detect droplets in mono- and polydisperse flow cell systems. The detection accuracy of each model shows excellent statistical symmetry with an implementation of the Hough transform as well as relevant ImageJ plugins. The models were successfully used as droplet detectors in non-microfluidic micrograph observations, where these data were not included in the training set. The models outperformed the traditional methods in more complex, porous-media simulating chip architectures with a significant speedup to per-frame analysis times. Implementing these neural networks as the primary detectors in these microfluidic systems not only makes the data pipelining more efficient, but opens the door for live detection and development of autonomous microfluidic experimental platforms.
\end{abstract}


Keywords Droplet microfluidics · Emulsion · Image analysis · Object detection · Convolutional neural network · Deep learning

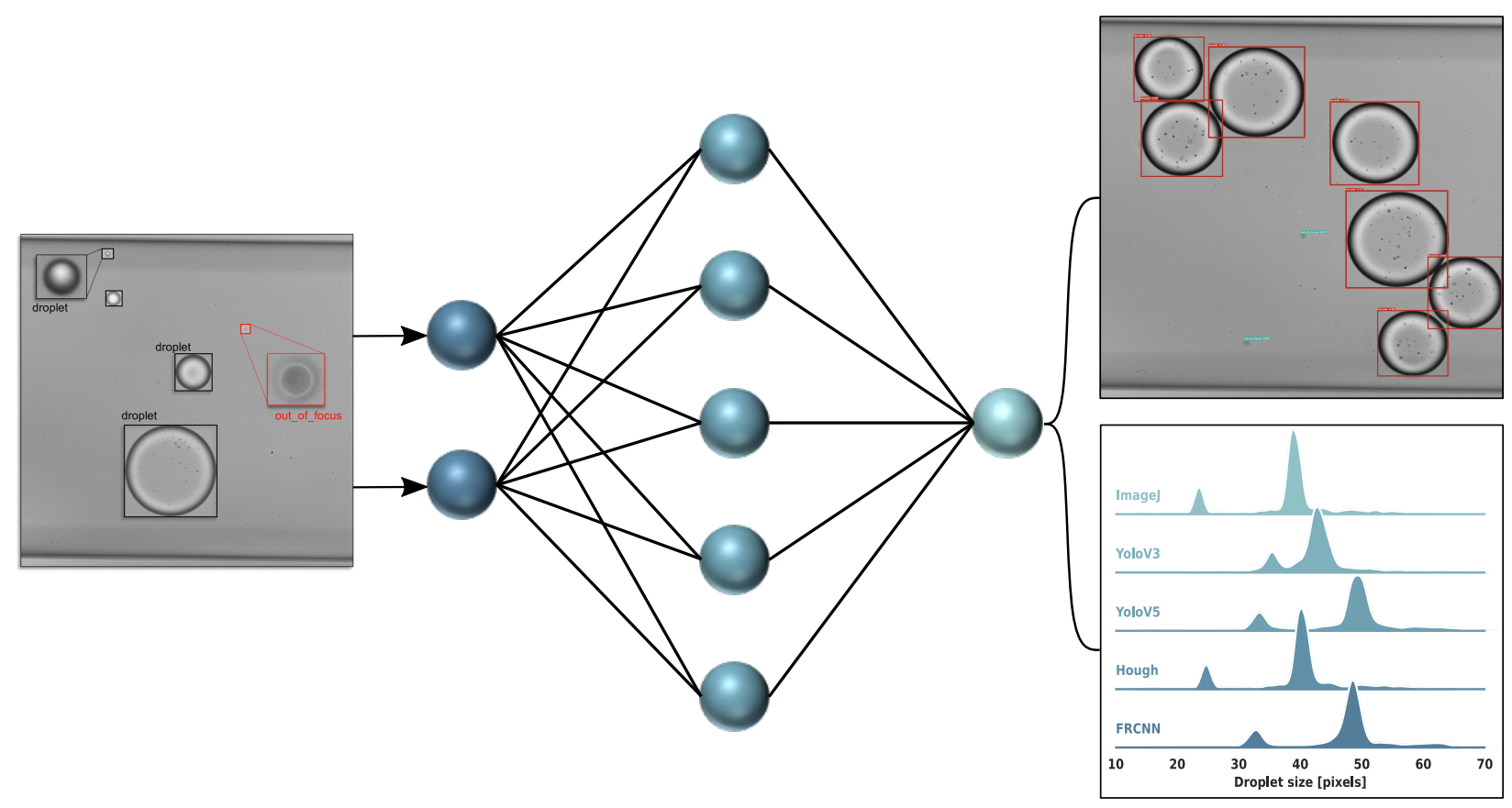

\section{Introduction}

Emulsions are kinetically-stabilized systems of certain liquids dispersed in others. Their stability and destabilization mechanisms are a crucial factor in a number of everyday products and industrial processes, such as food products, cosmetics, production of pharmaceuticals and wastewater treatment [1]. Destabilization most commonly occurs through coalescence and gravity separation, however other physicochemical processes such as Ostwald ripening or flocculation between droplets can also contribute to phase separation. Based on these stability processes, the continued research regarding measurement techniques which probe these phenomena is vital for ensuring development of the aforementioned systems and products. The physical tools which contribute to these techniques include include microscopy, turbidity measurements, light scattering, rheology, droplet manipulators, and more recently, microfluidics.

Within emulsion science, microfluidics is a new, but unsurprising addition to the experimental toolbox. Droplet-based microfluidics is a perfectly suited technique for studying emulsified systems due to its ability to precisely tailor and manipulate droplet parameters [2]. As far back as 2012, Bremond and Bibette [3] were able to identify microfluidics as a powerful tool for approaching emulsion science research (based on the steadily increasing number of reports produced yearly). Importantly, it enables work to be performed within a similar size scale common for industriallyproduced emulsions, but simultaneously reduces throughput volumes, thus significantly reducing waste produced during measurements. Furthermore, the inherent transparency of the microfluidic devices used in these studies facilitates visual observation of these phenomena, often via high-speed imaging. Therefore, classical image analysis has become a tandem subdiscipline that accounts for much of the harvestable data in these emulsified systems [4].

The inherent size of the flow channels in these microfluidic chips or devices $(<1 \mathrm{~mm})$, coupled to the velocity and number of droplets within an active system poses a challenge in regards to the technical specifications for visualization. Compared to steady-state microscopy, capturing the generation, flow and behaviour of hundreds or thousands of droplets per second often requires a high-speed camera operating in unison with the optics of a microscope. The definition which constitutes the baseline requirement for "high-speed imaging" is often vague and depends on the application, however in most cases it starts at the higher limit for standard microscopy imaging, usually 50-100 frames per second (fps). Most commercial high-speed cameras excel at recording greater than 1000 fps [5], and indeed we see that many reports within droplet microfluidics record within hundreds to thousands of fps $[6,7,8,9]$. Consequently, each experiment which could be comprised of a few seconds worth of video, results in thousands of frames that require processing and analyzing. Commonly, this analysis is performed via traditional software, plugins and scripts run on platforms such as ImageJ or Matlab, and less frequently other highly-specific, proprietary analysis programs provided by microscope and 
camera manufacturers. Regardless of analysis approach, the most commonly extracted features from these datasets are the number of objects or droplets, size (area, diameter), shape (aspect ratio, circularity) and position. The basic extractable parameters of these droplets enable even further calculation of system dynamics such as velocity or localized concentration of droplets.

Droplet feature extraction is enabled by implementation of standardized image processing techniques such as the Circle Hough Transform for example [10,11,12]. The digital image data produced by observation of microfluidic droplets is usually ideal, as the droplet interface presents in high contrast against the chip background and continuous phase fluid. The large degree of control over experimental conditions enables the production of high-quality image data where focus and lighting are tuned so feature extraction is usually successful in providing highly representative system data $[13,14]$. As experimentalists begin to develop more complex microfluidic systems, the image-analysis portion of the experimental pipeline begins to bottleneck the process, similar to other disciplines which rely on feature extraction [15]. Complex chip architectures usually include either connections to external electrical components or on-chip features which induce specific droplet transport mechanisms. The addition of these chip features directly impede feature extraction in software suites such as ImageJ or Matlab. As experimental complexity scales, the image analysis portion of the pipeline not only increases computational and analysis time, but also requires the experimentalist to dedicate significant effort to data filtration and thresholding to enable accurate feature extraction. This manual pre-processing impacts the statistical robustness of emulsion or droplet data in more exotic microfluidic systems. The impact of this necessitates the development of new, smarter analysis methodologies that preserve statistical robustness, while limiting involvement in curation and treatment of raw data.

In order to facilitate a break-away from traditional, yet bulky image analysis pipelines, deep-learning methods have the potential to excavate the entrenched nature of these problems via the intrinsic handling and representation of raw data used in the slow, orthodox analysis. This untreated data can be fed to a model, which learns the necessary representations to tractably approach the task of classification or detection in a well-defined system of objects. The representations of the data are systematically passed through a series of modules, which perform transformations into subsequently more abstract representations [16, 17]. Within this network of abstract representation, complex functions emerge from these simple, yet non-linear modules which serve the purpose of enabling feature discrimination and suppression of irrelevant relationships in the decorrelated latent space [17, 18, 19, 20]. By feeding an image to this network, the latent space can begin to construct representations for the presence of certain pixels which ultimately contribute the formation of a feature structure. Subsequent abstraction can then take these suspected features and arrange ensemble combinations which correspond to the target objects, that are reconstructed through the generative portion of the network [16, 17].

Since the emergence of deep neural networks (DNNs), there have been a number of machine- and deep-learning based tools developed for image analysis based on microscopy data, especially in the context of biological science. A prize application has been using these DNNs for time-intensive tasks such as cell detection and counting or even segmentation, and various model development has specifically tackled this [21, 22, 23]. Of these reports that used these DNNs for live cell imaging and eventual single-cell segmentation [21], the authors claim that this approach is not only more time efficient, but it also broke ground in regards to utilizing computer vision to analyze previously untenable co-culture experiments. Outside of singular instances where models were developed for specific tasks, entire analysis pipelines have been developed to handle end-to-end detection and analysis of objects common in microscopy such as cells $[24,25,22,26,27,28,29,30]$. For example, of these workflow packages, Suleymanova et al.[24] developed an open-source software (FindMyCells) built on implementing DNNs to accurately detect astrocytes in immunohistological images. Once again, the common claim from the authors is that by benchmarking specific DNNs against traditional methods, there is an attainable outperformance by the former, where it almost achieves superhuman detection and classification capabilities.

Biology and emulsion science naturally have overlap due to how both fields have been exploiting the advantages of the "lab-on-chip" aspect of microfluidics. There has been some research involving the integration of machine- and deep-learning methods into microfluidics which also investigate primarily cell imaging in flowing systems [31]. As opposed to pipelining steady state microscopy for cell imaging via a DNN, Heo et al. used a model which was able to classify flowing cells in real-time, opening the door for other integrations of a DNN other lab-on-chip applications, such as flow properties. Hadikhani et al. were able to extend the application of a DNN to beyond biological applications, to measure fluid properties of droplets flowing in a microfluidic channel [32]. They trained the network to accurately identify flow rate and mixture concentrations (water/isopropanol) of a dispersed phase based on the flow pattern of droplets. This enables extending these concepts to further exploit the efficiency of these DNN pipeline to generalized droplet detection and many more applications. Based on this, we posit that in the coming years machine- and deeplearning will become a major area of focus for microfluidic researchers due to the immense potential for developing smart analysis and control pipelines that outperform traditional methods. 
In this paper, we aim to demonstrate the viability of using various DNNs as tools for analyzing droplet based microfluidic data in the context of emulsion science. The detection of circular objects is a decades old computer vision task, inherently tied to microscopic observation, which has previously been universally approached by various implementations of the Hough transform or other computational solutions. As microfluidic architectures become more complex, the detection task also scales challenging traditional analysis methodologies by increasing the amount of resources necessary to shepherd data from beginning to end of pipeline. We show that the neural networks trained on custom microfluidic droplet data are robust and capable of universally handling the droplet detection task across various experimental systems and produce data which either rivals or outperforms traditional approaches, while significantly reducing user time involvement.

\section{Overview of Convolutional Neural Networks}

To fully leverage this deep, representative treatment of data and the subsequent generative detection or classification via DNNs, the convolutional neural network $(\mathrm{CNN})$ has emerged as the ubiquitous architecture for approaching various dimensional object detection problems across many scales of complexity [33,34]. Here we briefly summarize the generalized architecture and mathematical foundation for training and applying CNNs to object detection [17, 34, 35].

Once the image input is decomposed into a tensor and introduced to the CNN, each layer the represented data passes through is known as a feature map. As the tensor input traverses prior and subsequent neurons of the CNN, the data is filtered via a convolution operation which extracts localized features, and the assigned weights are adjusted so the model output eventually converges to an expected output. In order to fully construct the feature map at each step of the $\mathrm{CNN}$, a non-linear function, such as the rectified linear unit (ReLU) is utilized as the decision maker in obtaining the final feature maps for the corresponding neuron. In post-convolution neurons, specifically after the application of a non-linearity, a secondary operation known as pooling can be applied which down-samples or reduces the size of the feature maps in order to summarize feature extraction. Cyclical input between neurons, starting at convolution and retention of filter weights to non-linear boundary application and down-sampling, will eventually yield a representation which has sufficient lower dimensionality to be passed to a last component of the CNN. This final softmax step produces a generative vector output that could be a conditional class-based probability.

The retention of filter weights for the feature maps can be exploited by training the neural network to perform classification or object detection. The instructions for the navigation of feature maps in certain, well-defined input spaces can be obtained through the optimization of a cost or loss function via stochastic gradient descent (SGD). This minimization relies on querying the model to output a prediction, which then iteratively attempts to reduce the distance between the prediction error and ground truth. Upon being asked to produce the same prediction, weights are retained which allow the eventual convergence of this error based on the cost function. The key concept of SGD is that there is an overall traversal to a better answer by eventually settling within a cost minima. In contrast to the "feed-forward" generative functionality of a CNN, SGD is performed from final layer to first. The gradient of the cost function feeds back into the final layer of the model, whose subsequent gradient acts on the layer before it, and so on via back-propagation $[17,36,37]$.

The SGD-based approach to training these models includes optimization of the hyperparameters that dictate the degree at which the cost function traverses the minima/maxima landscape. The eventual success or effectiveness of a model at certain tasks is dependent on the initialized weights, or some form of ansatz that shapes the weights-tuning [38]. The effectiveness of the weights can be further skewed with inefficient hyperparameters such as the learning rate of the model, wherein rapid learning can mistakenly trap the cost function in local minima or in other cases promote aimless walking across barren plateaus [39]. Opposite to the overstepped minimization, is maintaining a training environment for long enough where the model is overtrained and could engage in overfitting. In this case, the result of future predictions could become unreliable [40]. Developing approaches to counteract these pitfalls and developing better performing models is a highly active ongoing area of research $[17,34,41]$.

In this work we leverage two implementations of anchor-based object detection CNNs, which are considered current state of the art models, that both approach the generative portion of the data pipeline differently. The original demonstrations of these two CNNs are: Faster R-CNN [42] and YOLO [43]. Below, we briefly discuss the models and show the generalized visualizations of the architectures in Figure 1.

\subsection{Faster R-CNN}

Faster R-CNN is one of the current, penultimate innovations on a series of CNNs (R-CNN[33], Fast R-CNN[44]) which all approach the object detection problem via region proposal. Generally with this approach, regions of interest (RoI) are generated after a coarse scan of the input space followed by the computation of feature maps which are eventually 
A)

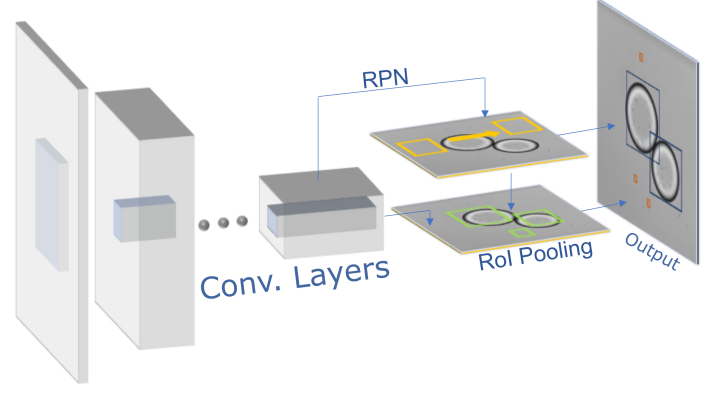

B)

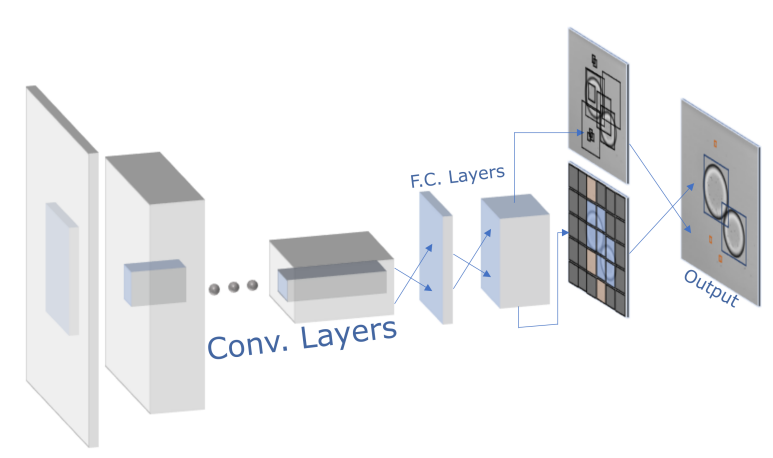

Figure 1: Brief visual representations of the models used in this work. A) Readapted grapical interpretation of the Faster R-CNN model. The first part of the model passes image input through a series of convolutional feature mapping layers. The backbone has a parallel region proposal network (RPN) grafted onto it which employs a sliding window that proposes regions of interest that are sent back into the pooling layer. The classification and bounding box regression loss proposals from the RPN are compounded to produce refined object classification and bounding box position tensors. B) Similarly reduced graphical interpretation of the YOLO architecture. Images are also passed to a series of convolutional feature mapping layers before being passed to the fully connected (F.C.) layers. The unified inference output is a result of simultaneous performing of detection and classification. For a more comprehensive exploration of the model architectures, we encourage referencing the original literature which first proposed these models [42, 43].

passed to the softmax portion which proposes a classification for these RoIs. The Fast R-CNN architecture builds on the previous model by not only proposing RoIs, but generating a bounding box regressor in addition to the softmax class probability. However, region proposal in these CNNs is an expensive task which bottlenecks efficiency. Ren et al. [42] then developed Faster R-CNN, one of the the models used in this work, which grafts an additional region proposal network (RPN) to the backbone of the CNN which shares the convolutional feature maps with the detection portion of the model. This parallel RPN simultaneously communicates with the traditional CNN backbone while sliding over the transformed convolutional feature map which produces a low dimensional vector. This is passed to the fully connected layers which handle the two pronged output of the box classification $(\mathrm{cls})$ and the eventual transformation of the box regression layer (reg) to bounding box coordinates . As the RPN slides across the input space, each window has several proposals for regions of interest which eventually become the bounding boxes for the detected object, where the number of maximum proposals is $k$. The resulting outputs are via the aforementioned reg and $c l s$ layers, where the former has $4 k$ outputs generating the coordinates of $k$ bounding boxes, and the latter handles $2 k$ estimates of probability of object $(o b j)$ or no object (noobj). The proposals are relative to the reference boxes, called anchors, which are centered at each window, yielding 9 total anchors at each window.

Anchor based proposals have an important implication in the alternative model used in this work and are discussed further. However, in the context of Faster R-CNN, the usage of anchors enables approaching convolution at multiple scales, or aspect ratios. Being able to to utilize the features maps via dynamic anchor scales is the key behind mitigating the majority of the computational cost associated with multi-scale input $[33,42,44]$. Furthermore, leveraging this approach to anchors at each interest region is key to the loss function involved in training and weight fine-tuning of Faster R-CNN. The reduction of class probability to the binary obj and noobj is directly associated to the highest degree of intersection-over-union (IoU) of the proposed anchor and the ground-truth bounding box. For higher degrees of IoU with ground-truth a positive label is assigned and vice-versa for lower degrees of IoU, and thus the minimization of the loss function is defined as [42]:

$$
L\left(\left\{p_{i}\right\},\left\{t_{i}\right\}\right)=\frac{1}{N_{c l s}} \sum_{i} L_{c l s}\left(p_{i}, p_{i}^{*}\right)+\lambda \frac{1}{N_{\text {reg }}} \sum_{i} p_{i}^{*} L_{r e g}\left(t_{i}, t_{i}^{*}\right)
$$

Where, $p_{i}$ is the prediction probability of some anchor, $i$ being an object, compared to the ground truth label $\hat{p}_{i}{ }^{*}$. The term $t_{i}$ stores the bounding box coordinates and its ground-truth counterpart $t_{i}{ }^{*}$ which addresses coordinate overlap in positive anchors. The loss term associated with the binary classification, $L_{c l s}$, of obj vs. noobj is a log loss while the bounding box regression loss, $L_{r e g}$ is a smoothed function to mitigate exploding gradients $[33,34,42]$. The outputs of the $c l s$ and reg layers are $\left\{t_{i}\right\}$ and $\left\{p_{i}\right\}$ which are normalized by $N_{c l s}$ and $N_{r e g}$ and finally shaped by the balancing parameter, $\lambda$. The loss function simultaneously tunes of all weights in an end-to-end way which eventually contributes to inference times of $5 \mathrm{fps}$ on typical workstation GPUs [42]. 


\subsection{You Only Look Once (YOLO)}

In contrast to interest region based detection and classification, an alternative architectural "reapproach" to the object detection problem is framing it as a purely regression task. Various prototypical approaches $[45,46,47,48,49,50]$ which tried to use this concept, attempted to address bottlenecks or speed issues with competing frameworks such as Faster R-CNN, but ultimately were hindered by limitations introduced in reducing the overall complexity of the architecture. Redmon et al. developed a model which eliminates the the general repurposing of classifiers as detectors and handles the bounding box regression and class probability in a single backbone where you only look once (YOLO) at full image inputs [43]. The single throughput architecture is composed of 24 convolutional layers followed by 2 fully connected layers culminating in a $7 \times 7 \times 30$ tensor output of bounding box predictions. The reduction of complexity in YOLO enables the direct prediction of bounding boxes and associated class probabilities which in turn can take and train on full images and perform the task in a unified manner. Task unification is achieved by discretizing full images into parceled $S x S$ grids which are then subsequently responsible for identification of potential object centroids within the corresponding grid cell. The bounding box predictions have an associated confidence which is defined as a product of probability $\operatorname{Pr}(\mathrm{Object})$ and IoU between the predicted box and the ground truth. Simultaneously, the grid cells also produce class probability, $\operatorname{Pr}\left(\mathrm{Class}_{i} \mid \mathrm{Object}\right)$ which are then used to produce class-specific confidences along with the coordinates of the predicted bounding boxes, as shown in:

$$
\operatorname{Pr}\left(\text { Class }_{i} \mid \text { Object }\right) * \operatorname{Pr}(\text { Object }) * I o U_{\text {pred }}^{\text {truth }}=\operatorname{Pr}\left(\text { Class }_{i}\right) * I o U_{\text {pred }}^{\text {truth }}
$$

The final layer of the model operates with normalized bounding box coordinates and utilizes a linear activation function. However, all other layers within the backbone use a leaky ReLU. The discretized grid based approach can sometimes result in a per grid confidence of zero which can skew the gradient thus diverging the training early [43]. Analagous to Faster R-CNN, when minimizing the loss function, two shape parameters are used to treat the weights where $\lambda_{\text {coord }}$ and $\lambda_{n o o b j}$ are used to increase loss from bounding box coordinate predictions and decrease loss from confidence predictions for null boxes. The multi-part loss function that is tuned during training of YOLO is:

$$
\begin{gathered}
\lambda_{\text {coord }} \sum_{i=0}^{S^{2}} \sum_{j=0}^{B} \mathbb{1}_{i j}^{o b j}\left[\left(x_{i}-\hat{x}_{i}\right)^{2}+\left(y_{i}-\hat{y}_{i}\right)^{2}\right] \\
+\lambda_{\text {coord }} \sum_{i=0}^{S^{2}} \sum_{j=0}^{B} \mathbb{1}_{i j}^{o b j}\left[\left(\sqrt{w_{i}}-\sqrt{\hat{w}_{i}}\right)^{2}+\left(\sqrt{h_{i}}-\sqrt{\hat{h}_{i}}\right)^{2}\right] \\
+\sum_{i=0}^{S^{2}} \sum_{j=0}^{B} \mathbb{1}_{i j}^{o b j}\left(C_{i}-\hat{C}_{i}\right)^{2} \\
+\lambda_{\text {noobj }} \sum_{i=0}^{S^{2}} \sum_{j=0}^{B} \mathbb{1}_{i j}^{\text {noobj }}\left(C_{i}-\hat{C}_{i}\right)^{2} \\
+\sum_{i=0}^{S^{2}} \mathbb{1}_{i}^{o b j} \sum_{c \in \text { classes }}\left(p_{i}(c)-\hat{p}_{i}(c)\right)^{2}
\end{gathered}
$$

where $\mathbb{1}_{i}^{o b j}$ and $\mathbb{1}_{i j}^{o b j}$ are denotions of, firstly, an object appearing in grid $i$ and secondly that the $j$ th bounding box predictor in cell $i$ is actually the one conducting the prediction. The aforementioned shape factors are involved when a penalization is applied, only if there is actually an object in cell $i$. In the discretized grid, the parametrized bounding boxes are composed of centroidal coordinates which are denoted by $x_{i}$ and $y_{i}$ as well as their corresponding ground truths, $\hat{x}_{i}$ and $\hat{y}_{i}$ and the normalized bounds of the grid $\left(w_{i}\right.$ and $\left.h_{i}\right)$ and their ground truths $\left(\hat{w}_{i}\right.$ and $\left.\hat{h}_{i}\right)$, respectively. The final parameter in the training in the minimzation is the confidence, $C_{i}$. Due to the lightweight backbone and data pipelining, the original YOLO implementation was able to process images at $45 \mathrm{fps}$ and when further reduced to a "small YOLO" could push inference times of $155 \mathrm{fps}$. Later implementations of this model (used in this work) also make strides at increasing per frame inference. This inference speed is one of the reasons we chose this model as it unlocks the capability to eventually move analysis to a real-time video feed. 


\section{Methods}

\subsection{Microfluidic experiments}

The data in this paper was obtained from analyzing a mix of microfluidic image data from previously published papers as well as newly produced data to benchmark the models against traditional methods. Therefore the experimental methodologies used will only be discussed briefly and the reader is kindly referred to our prior published work for greater detail. The datasets for each specific type of analyzed phenomena were from the following sources: flow cells [51, 52] and coalescence events [53]. The analysis performed for droplet generation, polydisperse flow cells, micrographs and porous media is unpublished.

For all microfluidic experiments with flow, the liquid flow rate was controlled with low- and mid-pressure pumps (Cetoni $\mathrm{GmbH}$ ). The pumps were connected to a glass microfluidic chip, placed in a microfluidic chip holder (all fabricated by Micronit Microtechnologies) via PEEK or PFA tubing and FFKM ferrules.

All experiments were recorded with a high-speed camera (AX100 or WX100, Photron), connected to an inverted microscope (Ti/Ti2-U, Nikon). The microscope is equiped with a motorized stage (OptiScan III, Prior Scientific, UK) that is coupled to the camera via LabVIEW code. Experiments involving micromodels (porous media) and micrographs were imaged by obtaining a sequential continuous grid of images using the motorized microscope stage. Some basic parameters of all experiments are displayed in Table 1.

Emulsions for the micromodel experiments were generated using a droplet generation chip comprising of a T-junction. A disperser was also utilized for producing the emulsion for the polysdispersed flow cell experiment.

Table 1: Selected experimental details for various type of microfluidic measurements presented in this work.

\begin{tabular}{|c|c|c|c|c|c|c|}
\hline \multirow{2}{*}{ Experiment } & \multicolumn{2}{|c|}{ Continious phase } & \multicolumn{2}{|c|}{ Dispersed phase } & \multirow{2}{*}{$\begin{array}{c}\text { Frame rate } \\
\qquad 1 / s]\end{array}$} & \multirow{2}{*}{$\begin{array}{c}\text { Magnific } \\
\text { /px- } \mu \mathrm{m} \\
\text { ratio }\end{array}$} \\
\hline & Fluid & $\begin{array}{c}\text { Flow rate } \\
{[\mu \mathrm{l} / \mathrm{min}]}\end{array}$ & Fluid & $\begin{array}{l}\text { Flow rate } \\
{[\mu 1 / \mathrm{min}]}\end{array}$ & & \\
\hline Drop generation & MQ water & $50-250$ & $\begin{array}{c}\text { Xylene with } \\
0.1 \% \text { wt. } \\
\text { Span } 85\end{array}$ & 4 & 4000 & $10 \mathrm{X} / 0.50$ \\
\hline Flow cells (water) & $\begin{array}{c}3.5 \% \text { wt. } \\
\mathrm{NaCl}\end{array}$ & 160 & $\begin{array}{l}\text { Dodecane and } \\
\text { crude oils }\end{array}$ & $6-10$ & 8500 & $10 \mathrm{X} / 0.50$ \\
\hline Flow cells (oil) & Crude oil & 80 & $\begin{array}{c}3.5 \% \text { wt. } \\
\mathrm{NaCl}\end{array}$ & 5 & 4000 & $10 \mathrm{X} / 0.50$ \\
\hline $\begin{array}{l}\text { Flow cells (poly- } \\
\text { disperse) }\end{array}$ & $\begin{array}{c}1 \% \text { wt. } \mathrm{NaCl} \\
+450 \text { ppm } \\
\text { Tween } 20\end{array}$ & 15 & 1-Br-dodec. & - & 2000 & $15 X / 1.43$ \\
\hline Porous media & $\begin{array}{c}1 \% \text { wt. } \mathrm{NaCl} \\
+450 \text { ppm } \\
\text { Tween } 20\end{array}$ & 4 & 1-Br-dodec. & - & $\begin{array}{c}\text { single } \\
\text { snapshots }\end{array}$ & $10 \mathrm{X} / 0.95$ \\
\hline $\begin{array}{l}\text { Coalescence } \\
\text { events }\end{array}$ & $\begin{array}{l}3.5 \% \text { wt. } \\
\mathrm{NaCl}\end{array}$ & 160 & $\begin{array}{l}\text { Xylene with } \\
0.1 / 1.0 \% \text { wt. } \\
\text { Span85 }\end{array}$ & 6 & 13600 & $6 \mathrm{X} / 0.30$ \\
\hline
\end{tabular}

Two types of emulsions were used in the micrograph experiments: two monodispersed emulsions generated on the chip and two polydispresed emulsions prepared by means of the disperser. The monodispersed emulsions had dropet size of $45 \mu \mathrm{m}$ and $23 \mu \mathrm{m}$ magnification imaged at $10 \mathrm{X}(\mathrm{px}-\mu \mathrm{m}$ ratio $=0.95)$ and $15 \mathrm{X}(\mathrm{px}-\mu \mathrm{m}$ ratio $=1.43)$ magnification respectively. The polydispersed emulsions were referred to as coarse emulsion with mode droplet size of around $10 \mu \mathrm{m}$ $(10 \mathrm{X}$ magnification, $\mathrm{px}-\mu \mathrm{m}$ ratio $=0.95)$ and fine emulsion with mode droplet size of around $2 \mu \mathrm{m}(30 \mathrm{X}$ magnification, $\mathrm{px}-\mu \mathrm{m}$ ratio $=2.85)$. The used dispersed and conunious phases are the same as for the experiments with porous media.

\subsection{Image analysis}

\section{Reference image analysis}


Drop generation and flow cells. After importing to ImageJ, stacks of images were thresholded and converted to binary. In some cases, contrast and brightness had do be adjusted manually prior to thresholding. Later, the drop sizes and positions were extracted with the "Analyze Particles" feature with a filter (size $>10 \mu \mathrm{m}$ and circularity $>0.8$ ). For flow cells, this data was then exported to Matlab, where a custom script produced size distributions and coalescence frequencies. This procedure is described in greater detail elsewhere [51, 54].

Coalescence events. Image sequences underwent a similar procedure as described above, however with a lower circularity filter ( $>0.4$ ). In addition to areas, shape descriptors (aspect ratio, circularity, roundness) were additionally extracted. These were used to calculate the shape parameter, which allowed discrimination of standard, circular droplets from the coalescing objects. The procedure is described in greater detail in prior work [53].

Micrographs. First, the micrographs were denoised using ImageJ's non-local means denoising plugin (smoothing factor $=2$, sigma $=15$ ) [55]. Afterwards, the images underwent a three step processing: 1) binarization by threshholding (dark background), 2) application of the "Fill Holes" feature in ImageJ and 3) separation of touching droplets using the "Watershed" feature in ImageJ. The "Analyze Particles" function was used to obtain droplet number and sizes. The obtained data was processed and filtered in Matlab. The following filters were applied: 1) roundness $<0.85,2$ ) circularity $<0.8,3$ ) aspect ratio $>1.2$, and 4 ) diameter $<5$ pixels for polydispersed emulsions, and diameter $<10$ pixels for monodispersed emulsion.

Porous media. First, the images of the micromodel were binarized by thresholding (white background). Afterwards, "Analyze particles" was utilized to identify objects on the images, both droplets and pillars. The obtained data was filtered using a Matlab script: $38<$ diameter $<50$ pixels for monodispersed; $15<$ diameter $<90$ pixels for polydispersed. This filtering step allowed removal of data points representing pillars and satelite droplets from the dataset.

In cases when the Circular Hough Transform was used to detect droplets (flow cells [inlet], micrographs, porous media) the Matlab-native "imfindcircles" function was applied to the images using "Sensitivity" $=0.7-0.75$ and "EdgeThreshold" $=0.1$. For the experiments using micromodels the obtained data was filtered using Matlab script: $12<$ diameter $<50$ pixels for monodispersed and $10<$ diameter $<85$ pixels for polydispersed emulsions.

In one section (micrographs), NIS-Elements BR software was used to extract droplet counts. Native functions (thresholding and object count), as well as filtering through limiting size and circularity, were used to extract the number of droplets in all images.

\subsection{Neural Network Implementation}

The CNNs described above are publically available and three implementations were utilized in this work.

The Facebook AI Research group has maintained the repository which has been restructured under the Detectron2 umbrella which includes a model zoo for various CNNs including the Faster R-CNN version used in this work along with subsequent instance segmentation models such as Mask R-CNN [56]. The entire Github repository is available at facebookresearch/detectron2. The specific version of Faster R-CNN implemented here is the "Faster R-CNN X-101$32 \mathrm{x} 8 \mathrm{~d}-\mathrm{FPN}-3 \mathrm{x}$ " and for initializing the model, the pre-trained checkpoints for this same version were used to train our custom weights. The additional training parameters for this model were: 'image_batch_size $=4$ ', 'base_learning_rate of 0.001', 'warmup_iterations $=1000$ ', 'max_iterations $=4000$ ', 'solver_steps $=(1000,1500)$ ', 'gamma $=0.05$ '. The header parameters were: 'batch_size_per_image $=64$ ' and 'num_classes $=2$ '.

Two latter versions of YOLO were used in this work compared to the original Darknet implementation by Redmon $e t a l$. available here: pjreddie/darknet/wiki/YOLO:-Real-Time-Object-Detection. For the later versions of YOLO used in this work, we used YOLOv3 and YOLOv5 with more emphasis placed on the latter. YOLOv3 was mainly trained and deployed to compare against YOLOv5 which claims some of the weaknesses described earlier regarding identification of small and grouped objects were significantly improved upon. The YOLOv3 implementation was PyTorch based with training, validation and test data structured for the Keras API on the Darknet backbone (cloned and modified from: ultralytics/yolov3). To produce a fine-tuned set of weights for our custom microfluidic droplet data set, similar to Faster R-CNN, we initialized the model from pre-trained COCO weights available in the YOLOv3 repository. The following parameters were used to train YOLOv3: 'epochs $=500$ ', 'batch_size $=16$ ', 'img_size $=[640,640]$ ', 'learning_rate $=$ 0.001 ', momentum $=0.95$ ', 'decay $=0.001$ ' and 'num_classes $=2$ '. Number of classes across all datasets was 2 , albeit the classes are different across datasets.

Our approach to training YOLOv5 on the custom droplet dataset was similar to YOLOv3, but the entire implementation, including dataset structuring, was PyTorch based. Our model was cloned and modified from the following repository: ultralytics/yolov5 [57]. The training was initialized with pre-trained COCO weights available through the YOLOv5 repository and the configuration file specified to be trained was specifically the YOLOv5s version of the model. The training parameters used for YOLOv5 were as follows: 'epochs $=1000$ ', 'batch_size $=16$ ', 'image_size $=[416,416]$ ', 
'learning_rate $=0.001$ ', momentum $=0.95$ ', 'decay $=0.001$ ' and 'num_classes $=2$ '. The main difference with our training approach to this newer version of YOLO is extending training to 1000 epochs due to how quickly the model trains as well as reducing image input size to $416 \times 416$ pixels.

\subsection{Dataset Construction}

The final iteration of the generalized microfluidic droplet dataset used as the training and validation set at the time of writing was designed to represent a wide variety of droplets that could appear in a given two-phase microfluidic system. While being generally broad in terms of providing robust ground truth representations of droplets, we specifically excluded certain experiments which were then probed in our analysis where the droplet characteristics widely varied from the training set to investigate the inference breadth of the models. In other applied scenarios such as biological cell detection, the lighting conditions often heavily impact the detection metrics of a model $[21,23]$ and thus in terms of exposure we tried to maintain lighting consistency across our training set. Figure 2 shows examples of images which were annotated and compiled into the training/validation/test set.
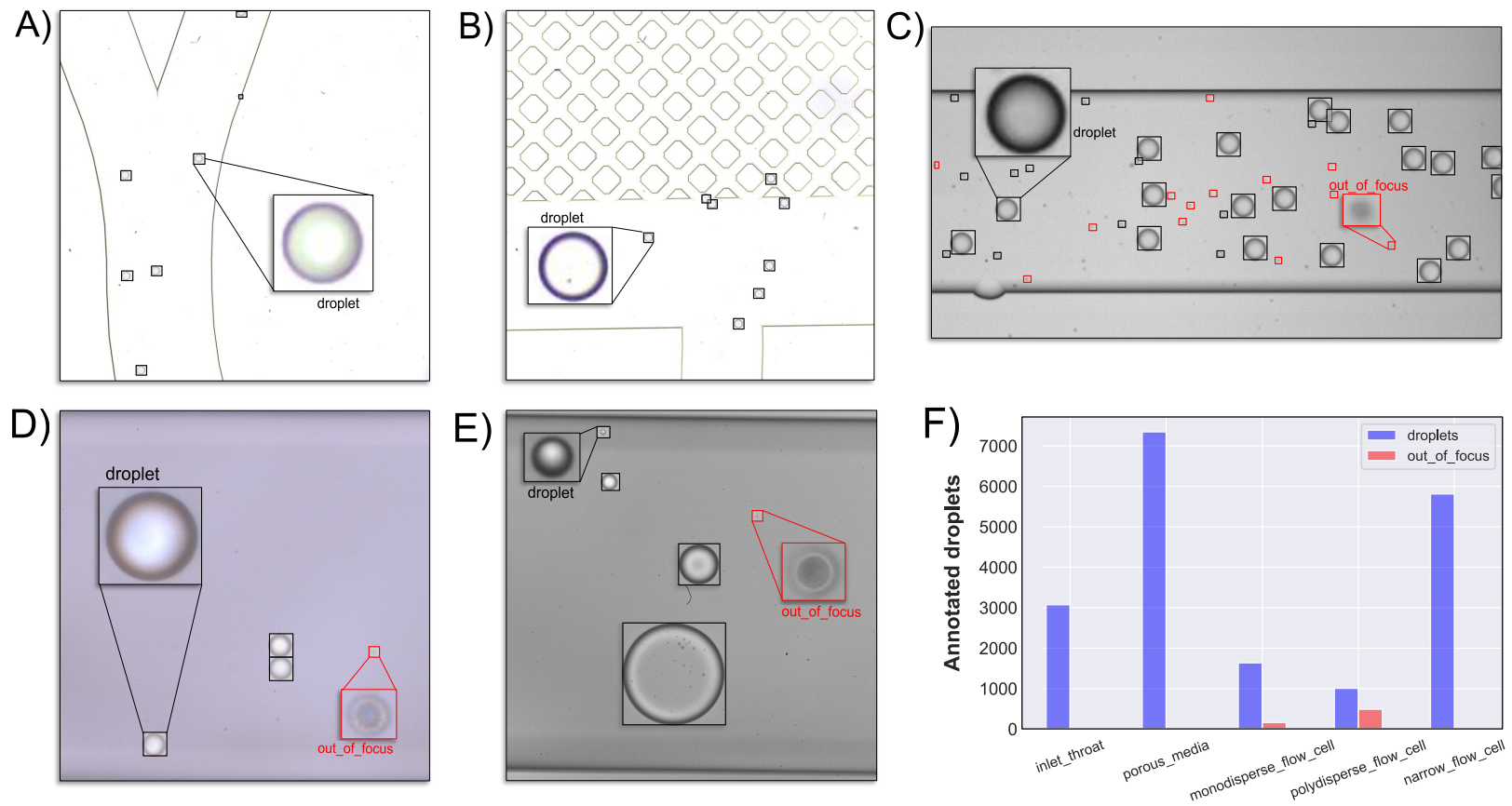

Figure 2: Shows an example of each type of image capture which was annotated and used in the general training/validation set. A) Inlet Throat, B) Porous Media, C) Narrow Flow Cell D) Monodisperse Flow Cell, E) Polydisperse Flow Cell. F) Also shown is a frequency distribution of droplets and a secondary "out_of_focus" droplet class in each of the image types in the generalized dataset.

A visual representation of how we annotated droplets from each microfluidic data subset is shown in Figure 2. Unlike other computer vision applications whose datasets may include greater than 50 classes [58] for applications such as self-driving vehicles, our general dataset is strictly limited to droplets and the occasional appearance of an out-of-focus droplet in chips with larger channel depths. In the general dataset shown in Figure 2, the biggest focus was to robustly represent the flow cell as it is the most common microfluidic tool to study various emulsified systems. In addition, we chose to represent a more complex detection task in the form of a porous media micromodel and the inlet throat. The chip architecture is well defined and presents a network of etch features that mimic the pixel ensembles which make up a droplet interface. The amount of images included from each experiment is as follows: 'Inlet Throat $=485$ ', 'Porous Media $=530$ ', 'Narrow Flow Cell $=148$ ', 'Monodisperse Flow Cell $=485$ ' and 'Polydisperse Flow Cell $=255$ '. The base amount of total images in the generalized dataset is 1903 without augementation. Out of 19,483 total annotated objects, out-of-focus droplets are very underrepresented in the generalized dataset at only 644 instances or 3.3\%. By including this class in the dataset, our intention was to identify poorly captured droplets and easily remove them from the harvested droplet data. 
The models were each trained on this dataset based on the parameters described in the implementation section (Section 3.3. The outcome of the training in regards to loss as a function of training iteration, for example, and other metrics such as mean average precision (mAP), recall are available in the supplementary information (Figures S1-S3). Confidence thresholds used during inference per experiment are also available in Table S1. After inference is performed, images are de-normalized by the native image resolution which allows extraction of bounding box coordinates and dimensions and transformation into micrometer values.

\subsubsection{PC specifications}

The workstation used for training and data analysis uses Windows 10, with 32 GB of DDR4 (3200 MHz) RAM (Corsair), Intel Core i9-10850K CPU running at 3.6 GHz and an Nvidia Geforce RTX 11 GB 2080Ti GPU.

\section{Results and Discussion}

\subsection{Droplet generation}

Outside of the broader systemic applications of microfluidics, the intrinsic physical mechanisms, such as droplet generation, can be exploited via various chip architectures, where T-junctions and flow focusing geometries are the most common. The size and number of generated droplets depends on the flow characteristics of both phases, but also the properties of the fluid [59]. Finding an optimal system with regards to drop size and generation frequency often requires several screening measurements, where various parameters are systematically tested [60, 61, 62]. Many reports in the literature also rely on specific drop size or monodispersity [63,64], where the presented method would also be applicable. Here we show the results of a simple experiment: droplets were generated and recorded during a step-wise increase in the flow rate of the continuous phase through a T-junction (Figure 3 ).

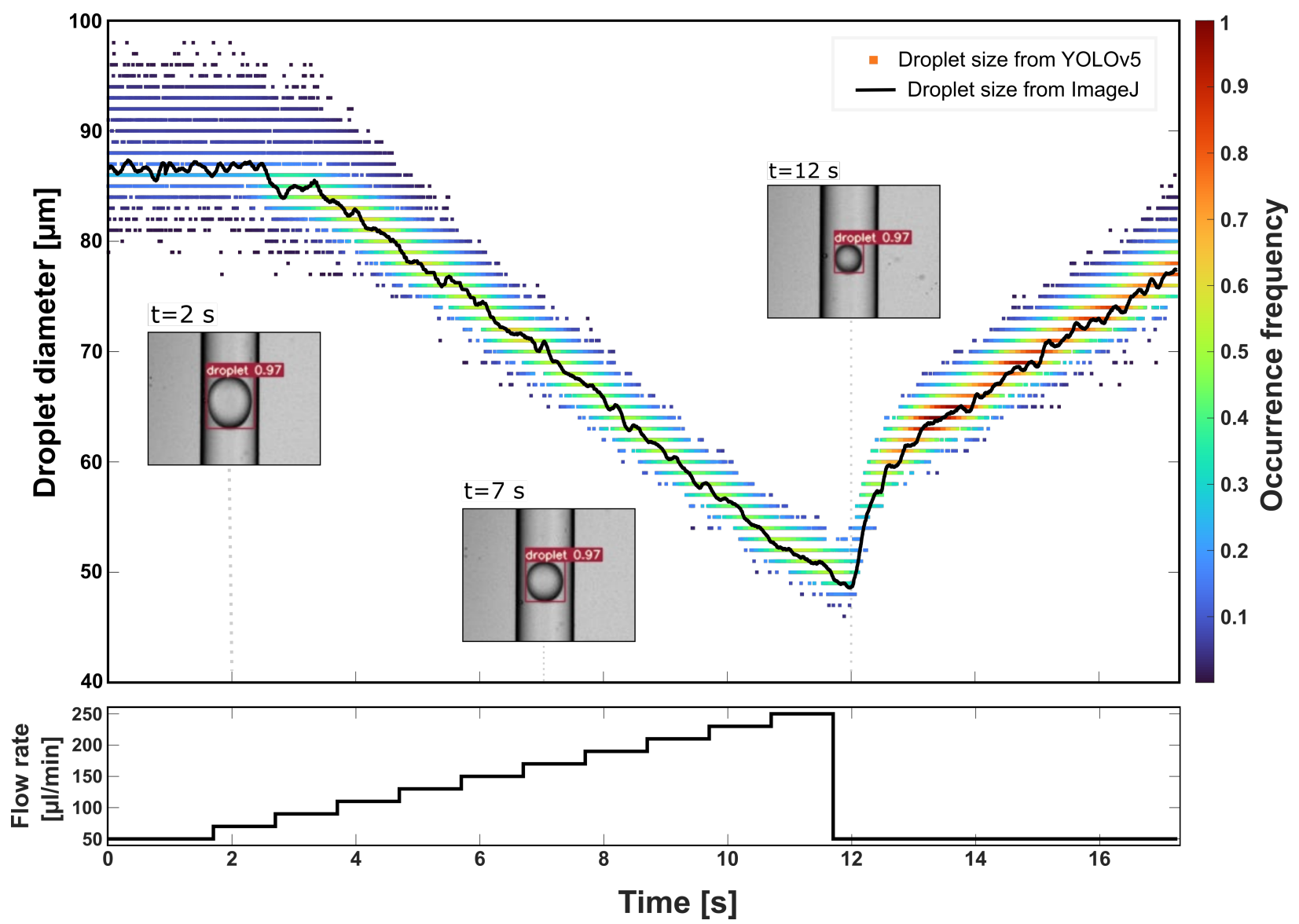

Figure 3: Droplet diameters obtained from ImageJ (solid line) and YOLOv5 (scattered points) for droplet generation experiment. The colour of the points corresponds to their relative occurance frequency in a given time unit. The line plot below shows the programmed changes in the flow rate of the continuous phase during the experiment. 
At time zero of the experiment, a constant continuous phase flow rate of $50 \mu \mathrm{l} / \mathrm{min}$ was introduced and maintained. After approximately 2 seconds, the continuous phase flow rate was ramped up by $20 \mu \mathrm{l} / \mathrm{min}$ per 1 second until reaching $250 \mu \mathrm{l} / \mathrm{min}$, at which point the flow was dropped back to the initial level, without ramping. The scattered points on Figure 3 are the values obtained via per-frame inference with YOLOv5. The colour scheme is a heat-mapping which represents the relative occurance of specific sizes in each time segment, i.e. dark and light blue-coloured points signify diameters that did not appear often in the dataset, while orange- and red-coloured points occured very frequently. The black line shows the data obtained from complementary analysis in ImageJ. For the sake of image clarity, we used a moving average to smooth out this data.

The outcome of this droplet generation stress test raises several points. In the first two seconds of the measurement, the detected sizes ranged from 80 to $95 \mu \mathrm{m}$. However, the mode in this segment oscillates around the "ground-truth", reference values obtained from ImageJ measurements. Inspection of the annotated images showed that the bounding boxes were quite often not-precisely fit to the droplets. One possible reason for that was the proximity of droplets to the walls of the channel. In these flow conditions (shown in the first image inset of Figure 3), the drops only had a few pixels worth of clearance in the horizontal direction. As flow rate is increased, expectedly the drop size decreases which also induced less fluctuation in the bounding box rendering. The precision deviation between droplet sizes corresponds to the proximity between the droplet interface and the edge features of the flow channel. The localized lighting conditions at the sub-5 $\mu \mathrm{m}$ scale could affect the model's ability to precisely discriminate between individual pixel ensembles belonging to the droplet or channel wall. A small lag in response between the change in the flow and the corresponding shift in drop size was observed, which could be attributed to the dead volumes in the tubing leading into the chip holder. At approximately $12 \mathrm{sec}$ of recording, the flow rate was brought back to the initial value of 50 $\mu \mathrm{l} / \mathrm{min}$. Consequently, the drop size started to increase, reaching approximately $80 \mu \mathrm{m}$ by the end of the recording. Interestingly, the diameter distribution was observed to be the narrowest in the last segment of the measurement.

Despite the oscillatory nature of YOLOv5's detection precision, both the average detected diameter and the mode in each time segment shared symmetry to detection performed in ImageJ. The fast ramping of the flow rate, combined with dead volumes in the system resulted in a delayed response in the drop sizes, meaning that the drop size at each flow rate is probably not the "steady state" diameter that should be expected in these conditions. Nevertheless, these measurements show that one can obtain reliable, reasonably distributed size data via YOLOv5 inference. Lastly, one important limitation of ImageJ should be noted here. The entire recording comprised almost 70,000 individual frames. Loading and analysis of these amounts of images on standard PCs or laptops in ImageJ is essentially intractable. Even on our analysis PC (specifications described in Section 3.4.1), just the loading all of the frames from memory into ImageJ consumes most of the RAM capabilites for more than $1 \mathrm{hr}$. In contrast, YOLOv5 took slightly longer than 10 minutes to entirely process and write detections for all 70,000 frames. Additionally, this lays the groundwork for various future applications where droplet-based systems in microfluidics are investigated in the context of using a CNN like YOLOv5, specifically capable of $>100 \mathrm{fps}$ inference (as in the experiment above). The model inference can be directly fed into a control algorithm which acts on the pumps to control flow for autonomous droplet or flow tailoring.

\subsection{Flow cells}

Subsequent to droplet generation in microfluidics, the various types of downstream droplet interaction are numerous and often the end goal of a microfluidic experiment, depending on the application. In most of our previous reports [51, 65], we focused on their coalescence or generally the interactions with other droplets. Here, we will showcase the analysis capabilities and symmetry to prior techniques using various CNNs on systems of freely flowing droplets, where the average diameter is smaller than the relatively large width (approximately $500 \mu \mathrm{m}$ ) of non-constrained flow cells. Our flow cell experiments can be divided into three subsets: 1) monodisperse droplets entering a wide channel shortly after generation (inlet of the coalescence chamber); 2) coalesced monodisperse droplets (outlet of the coalescence chamber); and 3) polydisperse droplets generated ex-situ and reinjected into a wide flow cell. Lastly, we will also present the detection of coalescence events during flow in microchannels. The droplets studied in each of these flow cell systems are shown in in Figure 4.

The first two (Figures 4A and 4B) were typically part of one experiment, where the inlet and outlet of the coalescence channel was recorded in order to count the droplets coming in, and then count/measure the size of the droplets flowing out of the channel. One important aspect of these experiments was that the projected area of the droplets was increasing proportionally to the number of coalescence events that they underwent. This made it feasible to segregate droplets into size classes and calculate how many coalescence events occured per time recorded. The data was then used to calculate the coalescence frequency, which is a common parameter to compare the stability of different emulsions [52, 66, 67]. In the contrasting case of polydisperse systems, the actual size distribution was of interest, as emulsions were prepared outside of the microfluidic chip and re-injected into the flow cell to record and later measure the droplet size. 

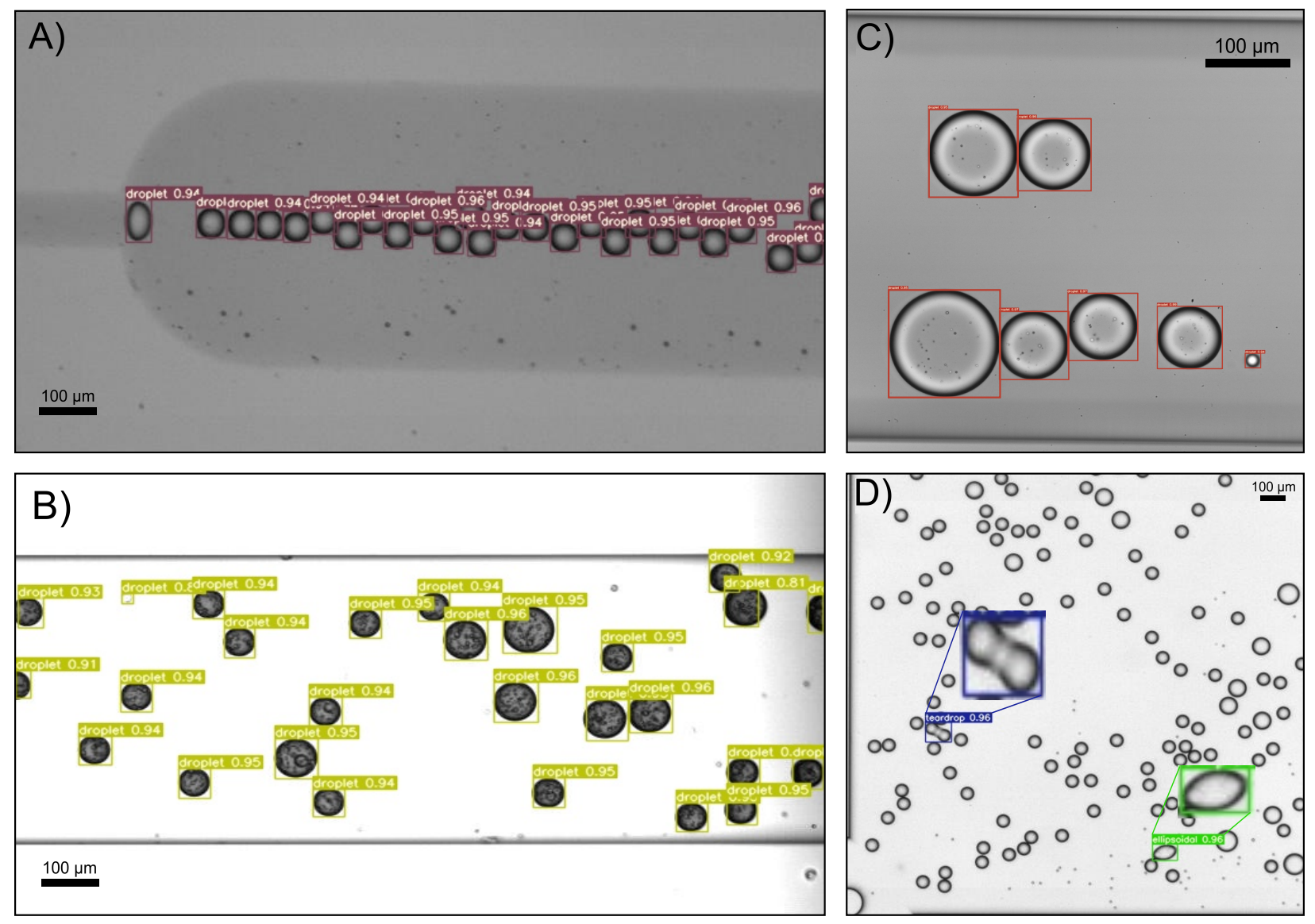

Figure 4: Inference performed by YOLOv5 on A) Monodispersed inlet, B) Coalesced monodisperse (contrast), C) Polydisperse flow cell, D) Alternately trained YOLOv5 detections of teardrop and ellipsoidal coalescence events. Insets are simply magnified contents of the inference bounding boxes.

\subsubsection{Monodisperse systems (inlet)}

After generation, the droplets or bubbles are often transferred to wider channels, where they can interact with each other $[9,68,69,70]$. The initial part of these coalescence channels allows to measure their size and count (or estimate) the number of generated droplets. Table 2 summarizes the data obtained for monodisperse droplets at the inlet of our coalescence channel. Here we compare the three CNNs with two commonly used approaches for droplet analysis in microfluidics: feature extraction via Matlab and ImageJ. All methods were tested against four sets of fluid systems. Three of them were water-continuous with varying contrast of the dispersed oil phase, while the last one was oil continuous, meaning that the background (continuous phase) was considerably darker. It should also be noted that here all analyses were performed on every 150th image in the frame sequences recorded for the flow cells. Our drop counting method relies on calculating drop velocity, which is needed to estimate the actual number of droplets passing through an RoI [54], rather than using the per frame appearance of all objects. Since the recording speed of the videos is on the order of thousands of fps (depending on the total flow rate), the same droplet is spotted several times as it passes through the detection window. By re-analyzing sets of filtered-out images, we could directly compare the detection efficiency of the CNNs and the overall analysis pipeline in contrast to the traditional methods.

Table 2: The number of detected monodispersed droplets using various methods.

\begin{tabular}{lccccc}
\hline Experiment & Matlab & ImageJ & Faster R-CNN & YOLOv3 & YOLOv5 \\
\hline OiW Model oil (weak contrast) & 1627 & 1559 & 1605 & 1591 & 1595 \\
OiW Crude oil (medium contrast) & 2983 & 2911 & 2957 & 2636 & 2931 \\
OiW Crude oil (strong contrast) & 2805 & 2749 & 2797 & 2678 & 2788 \\
WiO Crude oil (weak contrast) & 1548 & 1223 & 1503 & 1475 & 1446 \\
\hline
\end{tabular}


Here we see that overall, both Faster R-CNN and YOLOv5 are almost always matching the analysis outcome of ImageJ and Matlab. Based on the visual inspection of the annotated images, detection with Matlab's implementation of the Hough transfrom seemed to be the most precise, and indeed this method always yielded the highest counts of drops. The values obtained from both Faster R-CNN and YOLOv5 closely followed those of the classical Hough transform, as in most cases the deviation was not higher than 1-2\%. We noted however, that YOLOv3 detected significantly less drops in 3 out of 4 tested sets. The inlet recordings typically contain trains of droplets in contact with one another, typically in one to three parallel rows, depending on the system and flow rates used (see Figure 4A). When going through the inferenced images, visual verification showed that YOLOv3 failed to detect some droplets that were contained on the inner side of those droplet trains (as shown in Figure S4), consequently resulting in lower detection numbers. Interestingly, the oil-continuous system also yielded low droplet detections via ImageJ, compared to the complementary CNN analyses. Since the contrast between the oil and water phase is not as high as for the water-continuous systems, the thresholding of images in ImageJ was more demanding, and with small drop diameters, approximately $20 \%$ of objects were not detected.

\subsubsection{Monodisperse systems after coalescence (outlet)}

After passing through the coalescence chamber, many of the initially monodisperse droplets can merge together and re-emerge as larger droplets later in the flow cell. Since the area of the droplets increase proportionally to the number of coalescence events, it is common to use size classes instead of actual droplet sizes [71, 51]. The size classes are deteremined as follows: size class 1 is the the initial droplet size, size class 2 is the droplet formed after coalescence of two size class 1 dropslets, size class 3 is created by coalescence between size class 1 and 2 droplets, and so on. Figure 5 shows the droplet size class distribution from one of the experiments, where image analysis was performed with all three CNNs and ImageJ as a reference. Subsequent systems with similar analysis are available in Figure S5.

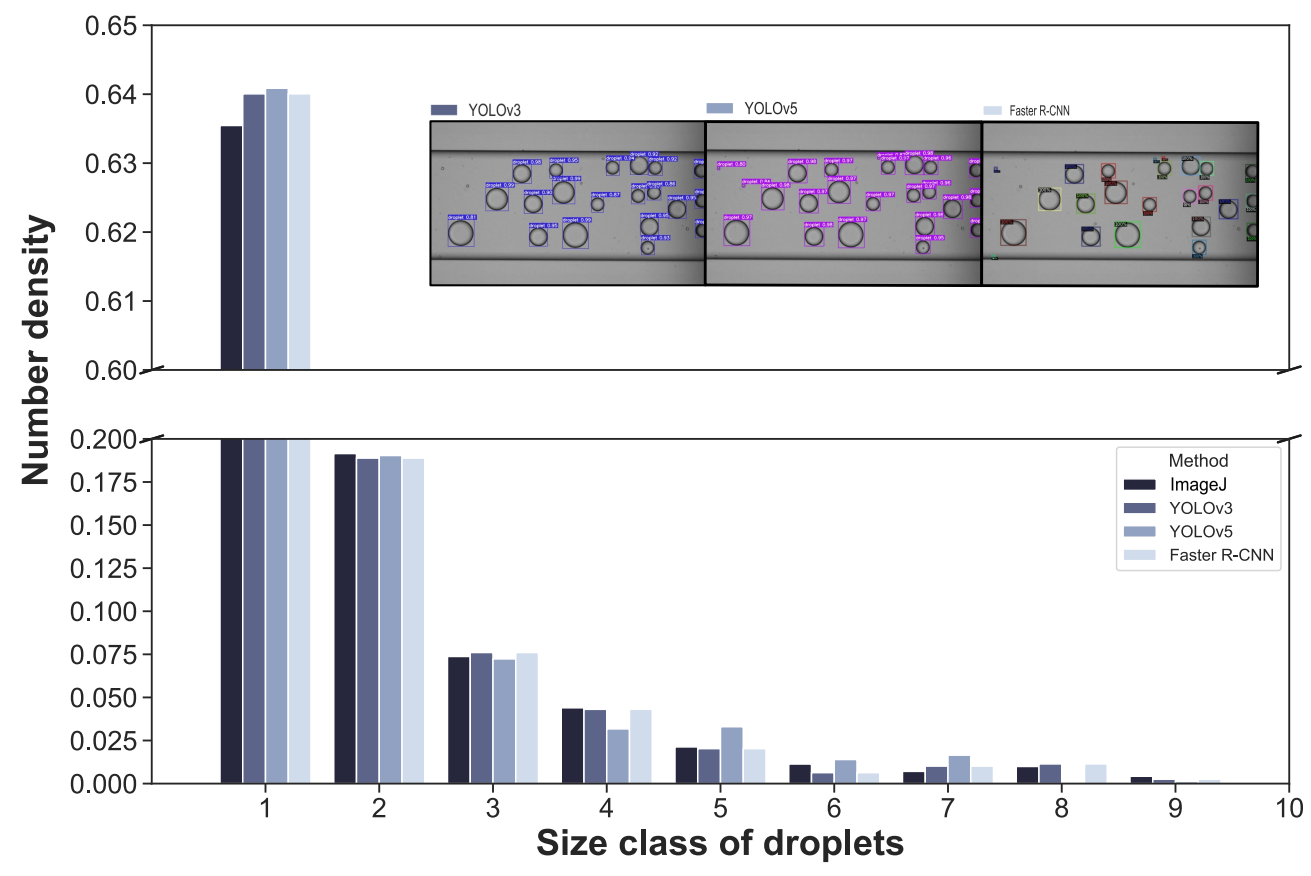

Figure 5: Size distribution of initially monodisperse droplets at the outlet of the coalescence chamber obtained with all three CNNs and ImageJ for reference. Data for OiW Model oil (weak contrast).

As in the previous RoI velocity calculations, ImageJ was asked to perform analysis on full sets of experiment frames, while the three CNNs were fed every 150th frame. This gave some discrepancy between the absolute drop counts, 
since the image filtering factor was only an approximation based on the velocity of the droplets. Therefore, all size distributions were normalized for more transparent comparison. The actual final processing of the data into discretized size classes was performed in Matlab based on the inference from each model and ImageJ. All analysis methods provide similar results, with some small deviations for YOLOv3 at droplet size classes 4 and larger. Even though the boundaries for all size classes were equivalent, various singular instances of droplets were assigned to different size classes, across the CNNs. This was most likely the result of bounding boxes providing a non-precise fit $( \pm 5 \mu \mathrm{m})$ to the detected objects, which in turn affected their size, and consequently the calculated areas of the droplets.

While informative, drop size class distributions are not very effective to compare the extent of merging in various systems. In most of our reporting on this type of droplet system, we tend to use coalescence frequency as a single-value parameter for comparison. Briefly, coalescence frequency is calculated by dividing the ratio of the number of droplets at the inlet and outlet by the residence time. It describes how often, on average, a single droplet undergoes coalescence event per unit time. Here, the analysis was also performed on selected systems with different merging characteristics (i.e. size distributions) and contrast between the continuous and dispersed phases (Table 3).

Table 3: Coalescence frequencies calculated from the droplet size distributions using various methods.

\begin{tabular}{lcccc}
\hline Experiment & ImageJ & Faster R-CNN & YOLOv3 & YOLOv5 \\
\hline OiW Model oil (weak contrast) & 2.112 & 2.067 & 2.021 & 2.067 \\
OiW Crude oil (medium contrast) & 0.760 & 0.752 & 0.735 & 0.755 \\
OiW Crude oil (strong contrast) & 0.436 & 0.414 & 0.410 & 0.379 \\
WiO Crude oil (weak contrast) & 0.552 & 0.704 & 0.694 & 0.705 \\
\hline
\end{tabular}

For this instance, we report three decimal points precision to probe if any significant deviation between analysis methods exists. In reality, the coalescence frequency values have to differ by ca. $20 \%$ minimum to observe significant differences between the systems, since the typical standard deviation between repeated measurements is typically not higher than $10 \%$. Consequently, all three CNNs and ImageJ provided nearly equivalent coalescence frequency values for the first two cases (weak and medium contrast), where YOLOv3 was weaker as it provided slightly lower values. The other two cases are more interesting and therefore worth discussing. In the water-continuous system with strong contrast, image analysis via the CNNs yielded significantly lower results. The system in question is shown in Figure 4B, with dark oil droplets presenting in a highly overexposed flow channel. Here, analysis with ImageJ was to some extent problematic, as it was difficult to properly threshold the images due to dark colour of the oil and inner (water) droplets. This led to lack of detection of some initial-sized drops, which slightly increased the calculated coalescence frequency parameter. While Faster R-CNN and YOLOv3 seemed to detect all the droplets in the images for this system and gave a similar coalescence frequency, a number of the larger droplets were not detected by YOLOv5 (potentially as a result of high confidence thresholding). This caused the frequency parameter to be lowered by an additional $10 \%$ compared to the other two models. One possible reason for the lack of detection could be the inner features of the droplets, as for all of the other systems YOLOv5 was working equally, if not better than the other models. Both Faster R-CNN and YOLOv3 (especially the former) detected many more satellite droplets or image aberrations resulting from the flowing fluids or the microfluidic channel, leading to false detections. These detections can be forced by YOLOv5 if inference is again performed with lower confidence thresholding. However, for the sake of the analysis, satellites and false detections were filtered out of the analysis set. In the oil-continuous system, all the models provided very similar values, but higher than the result obtained from ImageJ. As in the previous flow cells, ImageJ struggles in certain systems where there is poor contrast provided between the droplets and the background. Therefore, we expect that the data harvested by the CNNs is in fact more accurate and reliable for sensitive phenomena in monodisperse flow cell systems.

\subsubsection{Polydisperse systems}

Monodispersed systems are highly controlled model experiments which offer insight into many fundamental droplet interactions but rarely approach addressing emulsified systems with a realistic size distributions. Imaging of less rigidly controlled emulsions in a microfluidic flow cell poses image processing challenges that are not characterstic in monodispersed droplets. We included an annotated set of polydisperse droplets in the overall training set, seen in Figure 2, where various droplet morphologies and out-of-focus objects were prevalent. The inherent complexity of these polydisperse droplets increases especially in droplets with sizes greater than 100 pixels due to the high likelihood of droplets appearing within droplets - double emulsions. These very large droplets present at diameters sometimes 10 times greater than the smaller droplets. The goal of including this data was to teach the models that in polydisperse systems a droplet can exist at such varying levels of heterogeneous diameter, something that is unavailable in model monodisperse systems. At insufficient levels of training, precision as a function of training iterations has not converged 
enough with regards to being able to discern the interior droplets from the bulk droplet. However, as training iterations increase, with precise annotation, the models exhibit sufficient precision towards discerning double emulsions simply as whole droplets.

Additionally, when pivoting from narrow size distributions of droplets to emulsions with wide size distributions the focal plane becomes a factor which affects microscopic observation due to some of the droplets appearing out-of-focus (OOF). These droplets disappear fully or partially during the microsope observation of the flow and thus are omitted or inaccurately detected during image processing which contributes to statistical misrepresentation. Although, despite the models having the ability to identify out-of-focus droplets, the raw inference only visually distinguishes proposed bounds for the OOF droplet and thus a true size still cannot be ascertained. However, using DNNs for enhancement of images to super-resolutions is a highly researched area and could be a viable pathway for enhancing resolution for OOF droplets and investigating the frequency of their occurence visually and the potential effect on the droplet statistics [72].

We performed a flow cell experiment where a polydisperse emulsion was generated off-chip and then re-injected in order to generate a high fps dataset to be fed to the traditional analysis tools as well as the models. In contrast to the monodisperse droplets where we fabricate size classes for relatively narrow distributions, the measured hetergeneous sizes can be represented as true size distributions. In this dataset we used pixel values to highlight the limits of each technique, regardless of the actual droplet size. The per-method inference visualization as well as the corresponding size distributions are shown in Figure 6.

The outcome of the analysis here shows excellent statistical symmetry, specifically, between processing performed in ImageJ and having YOLOv5 and Faster R-CNN infer the bounding boxes on the polydisperse droplets. ImageJ struggles with the smallest droplets that appear in this flow experiment while YOLOv5 and Faster R-CNN are able to identify an order of magnitude higher. These small detections make up the bulk of the sample sizes depicted in Figure 6. YOLOv5 excels at detecting all small droplets $<10$ pixels and is still able to discriminate and classify non-usable OOF droplets. Faster R-CNN struggles with this which is why there are generally more droplets towards the small end of the total detections as it includes them into the results. YOLOv3 on the other hand is not a viable method, as it has up to this point struggled with very small object clusters and now also completely fails at detecting very large droplets and double emulsions. The detections of this model are still acceptable for droplets within 35-200 pixels but outside of this range, YOLOv5 and Faster R-CNN outperform the older counterpart of the former.

Based on general histograms binned every 10 pixels, we wanted to examine the precision of the models at a more granular level of inspection between these sizes (mid-to-large droplets), without the skew from the small droplet sizes at the tail of the distributions. Figure $6 \mathrm{E}$ shows the precision variation in terms of detection frequency as a function of droplet size for ImageJ, Faster R-CNN and YOLOv5. YOLOv3 is specifically omitted because of the poor detections of large droplets. Faster R-CNN is generally the model which reports more droplets from 100 pixels to approximately 400 pixels. All three methods converge well towards the extremely large sizes. The variation in frequencies between bins is best described by the spillover caused by imprecise bounding boxes. The same droplet which is detected on a per-frame basis in some cases could oscillate between the size bins as it travels in the flow channel based on the bounding box being rendered with imprecision between the box and the droplet interface. This precision oscillation when sequential frames are analyzed by the models is similar to what we observed in the droplet generation experiments in Figure 3, which also relied on a very large dataset.

\subsubsection{Coalescence events}

In addition to just droplet detection, we show that the models can be trained to discriminate inter-droplet interaction morphologies, such as various coalescence events instead of the actual droplets themselves. Measurement of coalescence time is an important topic in the literature concerning emulsions, and several microfluidic techniques have been reported previously [71, 73, 74, 75]. In our previous work [53], we have also reported a microfluidic method for measuring coalescence time. We define coalescence time as the time needed for the thin film, formed between two droplets in contact, to break. The coalescence time measurement was based on the detection of droplets in the process of coalescing, i.e. right after the moment of thin film breaking between the two drop interfaces, when the merged droplet returns to a typical, circular shape (see Figure 4D). The detection of these droplet deformations was the starting point for our calculation of the coalescence time. With the help of several shape descriptors available through ImageJ particle analysis feature, it was possible to calculate a shape parameter for all the objects in the recording. As a result, out of approximately 50000 frames and typically more than a million detected objects, one could filter out between 600 and 1000 coalescing droplets which were further analyzed. The image analysis performed in Image J is time consuming, due to the number of frames and size of the datasets, as previously highlighted.

Consequently, a new training set for YOLOv5 was prepared to enable a more precise and robust detection of coalescing droplets. We trained YOLOv5 on annotations which consisted of two morphologies of coalescence events: teardrop and ellipsoidal merging events. The a priori intention of developing weights for this detection task was to probe the ability of 

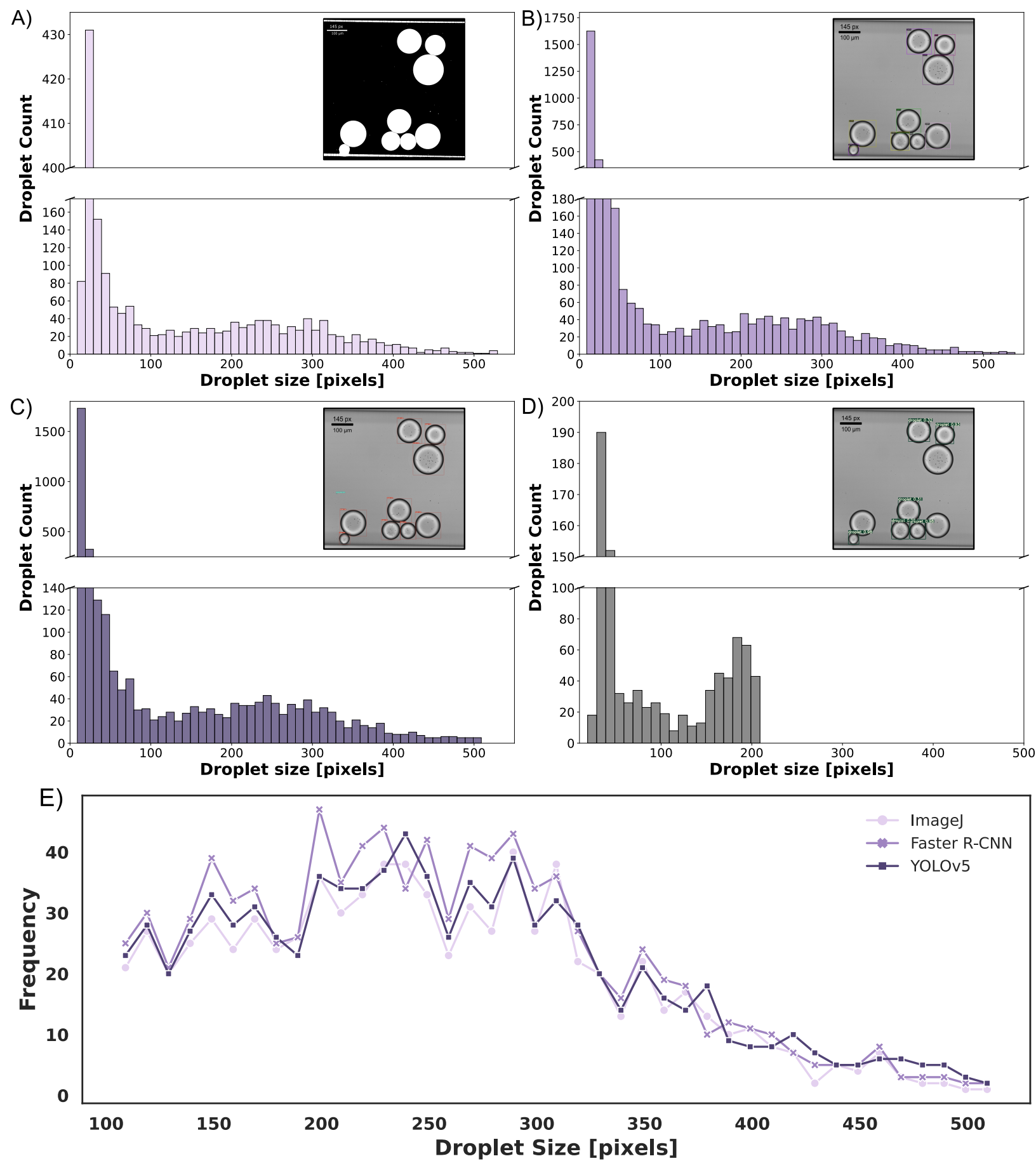

Figure 6: Droplet size distributions for a polydisperse emulsion observed in a flow cell. Each histogram shows the results of the detection and analysis for each method, A) ImageJ, B) Faster R-CNN, C) YOLOv5, D) YOLOv3. The inset of the upper secondary axis of each plot shows the results of the inference on the same frame from the experiment. E) Detection frequency for each binned droplet size for ImageJ, Faster R-CNN and YOLOv5. YOLOv3 is not included due to insufficient detections of larger droplets. 
the models to discriminate between droplet subclasses and not the actual droplets themselves. The training/validation/test (70/20/10 \% split) dataset for these coalescence events consisted of 786 images. The contributions from the each coalescence class consisted of 566 annotations for ellipsoidal events and 228 from teardrop shaped events. The model was trained with the same YOLOv5 parameters as the general droplet dataset described in Section 3.3. An example of inference performed by YOLOv5 is shown in Figure 4D, where an ellipsoidal and teardrop coalescence event occur on one frame. The number of detected coalescence events analyzed with YOLOv5 and ImageJ is presented in Table 4. Here, we show data for three different water-continuous systems, all with xylene droplets containing a nonionic surfactant (Span85) and at different temperatures (room or elevated temp.).

Table 4: The number of detected coalescence events in different systems using ImageJ and YoloV5

\begin{tabular}{cccc}
\hline Method & $0.1 \%$ wt. Span85 @2 $1^{\circ} \mathrm{C}$ & $1.0 \%$ wt. Span85 @2 $1^{\circ} \mathrm{C}$ & $1.0 \%$ wt. Span85 @ $40^{\circ} \mathrm{C}$ \\
\hline ImageJ & 886 & 725 & 878 \\
YOLOv5 & 1068 & 904 & 1047 \\
Same frame detections & 798 & 600 & 382 \\
\hline
\end{tabular}

Depending on the parameters of the experiment, different numbers of coalescence events were detected. When increasing the surfactant concentration, the droplets were more stable and consequently less merging events were observed. Upon increasing the temperature, coalescence improved, which is to be expected as thermal treatment of emulsions is one of the common methods of inducing oil-water separation. In regards to the number of the detected coalescence events between the different methods, in all systems there was an approximate 20-25\% increase in events observed by YOLOv5. It should be noted that both datasets were processed in the same way as described in our previous work [53], i.e. only events from frame 2000 and above were included and the events from consecutive frames were removed. This data filtration had a larger effect on the results from YOLOv5, where often one coalescence event was detected over several frames due to deformation between droplets.

Table 4 also lists the number of same frame detections for both methods. Here, we compared the frame numbers associated with a detected coalescence event reported by both ImageJ and YoloV5, and noted where the detections from both methods coincided. In the measurements at room temperature, these values are very comparable to the instances reported by ImageJ (ca. 100 lower for both systems), which means that YOLOv5 detected most of the same droplet merging events as ImageJ. The values for the higher temperature experiment are less symmetrical, however. One possible reason for that was that YOLOv5 was trained on two types of coalescence events (Figure 4D), namely teardrop, where the droplets are caught in the moment of the film breakage; and ellipsoidal, where two drops are already in the process of merging and returning back to circular shape. Image analysis via ImageJ in most cases did not allow for detecting teardrop events, as after thresholding the boundary of the two coalescing droplets was discontinuous, and therefore omitted in the detection process. At higher temperature tests there seemed to be significantly more teardrop events detected with YoloV5 (ca. 60\% of total amount, compared to ca. 30\% for the other two experiments at room temperature). Consequently, the same coalescence events could have been detected by ImageJ 2-3 frames after the detection with YOLOv5. And while the difference is quite small (few hundrends $\mu$ s), this could also potentially lead to more accurate coalescence time measurements and eventually on-line measurements due to high inference speeds of YOLOv5.

\subsection{Micrographs}

Thus far, the entirety of the droplet detection task has been framed in a microfluidics context. The models have been trained on purely microfluidic droplet data and then redeployed as analysis vehicles in other microfluidic systems. However, the dynamics regarding emulsion characteristics are often investigated via simpler avenues such as visual inspection after drop casting onto standard microscope slides [76]. Extracting data from these micrographs becomes a highly time-intensive process due to the image preprocessing and pipelining to commercial software or traditional analysis techniques, such as those discussed above. Hundreds of slices can be harvested from one drop-casted slide which can then yield greater than 50,000 detectable droplets, depending on the characteristics of the emulsion. Both approaches have drawbacks regarding pipeline efficiency and thus we posit that the acquisition speed of an automated stage coupled to the droplet detection models will not only allow for a significant increase in data acquisition rate but enable real-time acquisition. This is similar to a biological-focused implementation of Faster R-CNN and YOLO which used an automated stage that passed the microscope feed to the models which were trained to render detections of specific cells [23]. To implement this approach for emulsions on micrographs, single images can be simply passed to a persistent detection script or via batch processing. Once detection is invoked, of the three models used, Faster R-CNN has been shown to be the slowest while YOLOv3 and v5 inference speeds are less than $20 \mathrm{~ms}$ per frame. For YOLOv5, 
tuned with our weights, we reiterate that inference is typically $10 \mathrm{~ms}$ or less per frame on a GPU, and thus any potential bottlenecks will be related to the stage movement and subsequent image acquisition.

Nonetheless, in terms of the actual droplet detection, we found that inference on micrographs was actually a more complex task than detection in the other microfluidic systems investigated. Conditions outside of a microfluidic system are highly variable and the residence of droplets within the liquid film on the microscope slide can contribute to varying levels of focus in the acquired image. Additionally, outside of a microfluidic chip there is a high tendency for micro-scale contaminants to enter the system at large. These factors contribute to natural image augmentation which is not present in a raw microfluidic training set. These augementations coupled with the absence of annotated droplets coming from micrographs in the training set make detection more challenging for the models. Specifically, as in other CNN implementations for detection of microscopic objects, harsh lighting gradients often impact the inference capability of most models when fed raw images without prior image processing. This is evident as we benchmarked the CNNs against the traditional or commercial software on two monodisperse emulsions captured on micrographs with heavy vignetting. Table 5 shows the results of the total amount of droplets detected for each method and model. The vignetted micrographs for the 23 and $45 \mu \mathrm{m}$ emulsions are available in supplementary Figure S6, which also shows the inferred droplet bounding boxes for each $\mathrm{CNN}$.

Table 5: The number of detected monodispersed droplets using various methods.

\begin{tabular}{ccccccc}
\hline Method & Matlab & ImageJ & NIS-Elements BR & Faster R-CNN & YOLOv3 & YOLOv5 \\
\hline $23 \mu$ m droplets & 2638 & 2422 & 2375 & 700 & 1804 & 2100 \\
$45 \mu$ m droplets & 1397 & 1223 & 1170 & 700 & 1360 & 1431 \\
\hline
\end{tabular}

The variability seen across these methods indicates that lighting conditions significantly affect droplet counting especially in the vignette fringes. Specifically, in the Matlab implementation of the circular Hough transform, despite the monodispersity of the emulsions, the shaded fringe droplets are detected but assigned an incorrect diameter which contributes to skewing of the known size distribution. In the $45 \mu \mathrm{m}$ emulsion satellite droplets exist, which are also identified by the Hough transform and require filtering. YOLOv5 is the only of the models which also detects satellite droplets in addition to the intended droplets while YOLOv3 completely fails to identify the $<10$ pixel objects. Faster $\mathrm{R}-\mathrm{CNN}$ is the weakest of the models as it only detects droplets in which it is only near $100 \%$ confidence without any thresholding applied during inference. Although, YOLOv5 had the most success in droplet identification and construction of a homogeneous size distribution, we also tested these natural micrograph augmentations in more heterogeneous droplet distributions, fine and coarse. We considered the first fine because the mode droplet diameter was approximately $2 \mu \mathrm{m}$. The second of the heterogeneous emulsions had mode diameter of approximately $10 \mu \mathrm{m}$ which contributed to its coarseness. Figure 7 shows the droplet size distributions for these emulsions characterized by heavily pre-processed images in ImageJ, as well as raw data inference performed by Faster R-CNN, YOLOv3 and YOLOv5.

Immediately, similarly to the microfluidic systems, there is statistical symmetry between the distributions produced by our traditional control method and mainly YOLOv5 in both emulsions. Despite the fine emulsion tending to be slightly more homogeneously dispersed, the dispersity is broad enough where there is variance between the software and the models when dealing with smaller droplets. In both emulsions, the distributions converge well at droplet diameters around $10 \mu \mathrm{m}$. However, the task is more challenging for the predecessor YOLOv3 and the region-based Faster R-CNN. Frequency counts suffer and the smallest droplets are completely omitted even at very low confidence thresholds $(<50 \%)$ set during inference. In the case of finer droplets outside of microfluidic systems YOLOv5 is the superior choice for smaller, less familiar objects. As the droplet sizes grow along with a higher degree of polydispersity, the detection becomes similarly tractable for all three models where detection overlap is very high between ImageJ, YOLOv5 and Faster R-CNN. YOLOv3 still struggles due to the inherent architectural weaknesses which directly affects its inference capability for ultra-small objects (sub 10 pixels). The treatment of feature maps in YOLOv5 has been improved compared to older versions which still uses low resolution feature maps that contribute to YOLOv3's inability to detect ultra-small objects [57].

The original intention with constructing a generalized droplet detection dataset was that it could broadly tune several CNN architectures for ubiquitous droplet detection across many systems. Clearly, as the detection task evolves to droplet systems outside the microfluidic realm, the natural augmentation and and intrusion of disruptions to image quality suggest that the training task for both YOLOv5 and Faster R-CNN requires modification of the training data. Droplets are currently similar enough that the existing training set can tune the model to harvest droplet information with a competitive or better efficiency with significantly higher data acquisition rates (hours to seconds). Although we posit that higher degrees of precision can be achieved via inclusion of annotated micrographs in the model training or by assembling a training and validation set specifically for micrographs. Ultimately, the models struggle with 


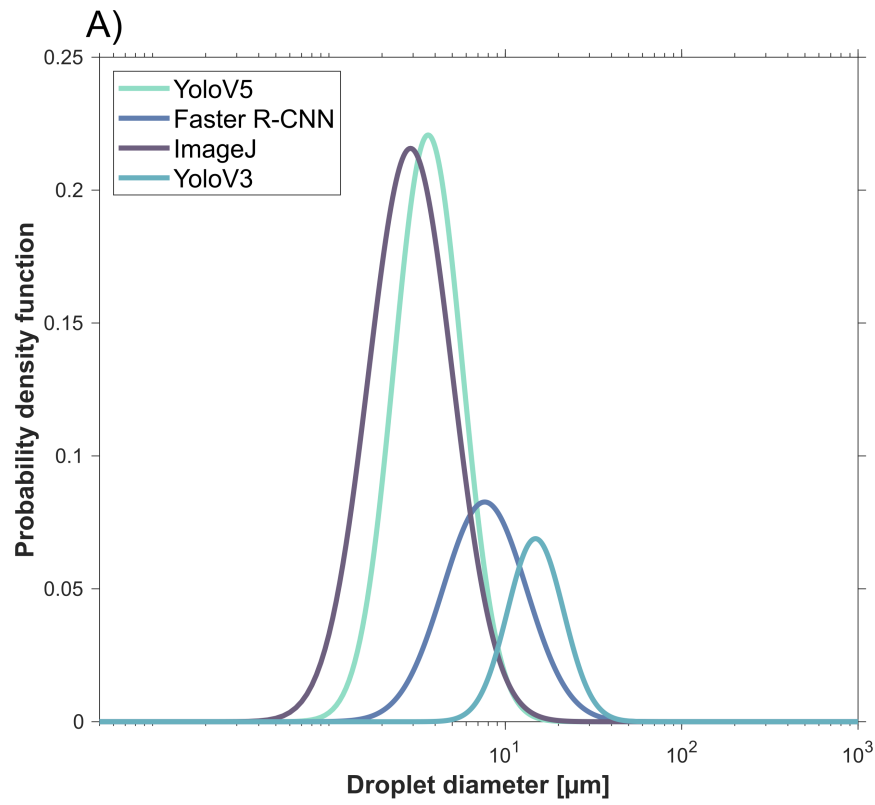

B)

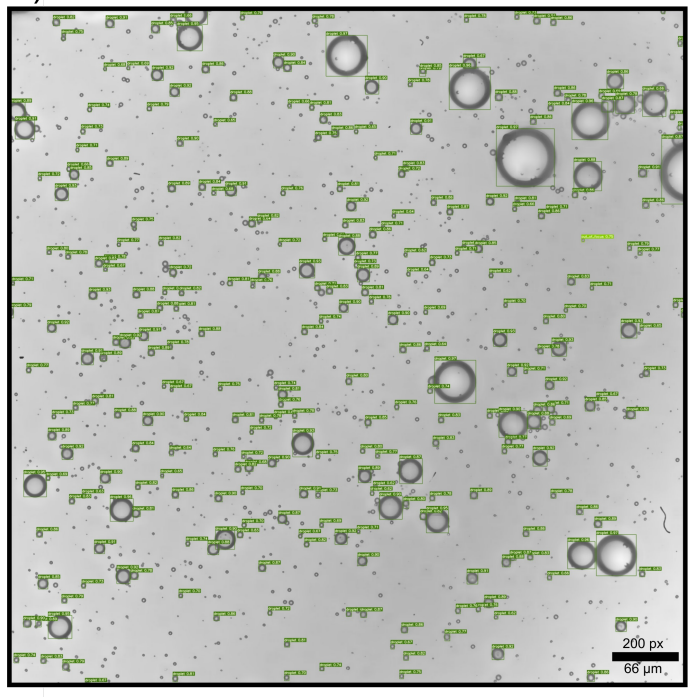

C)

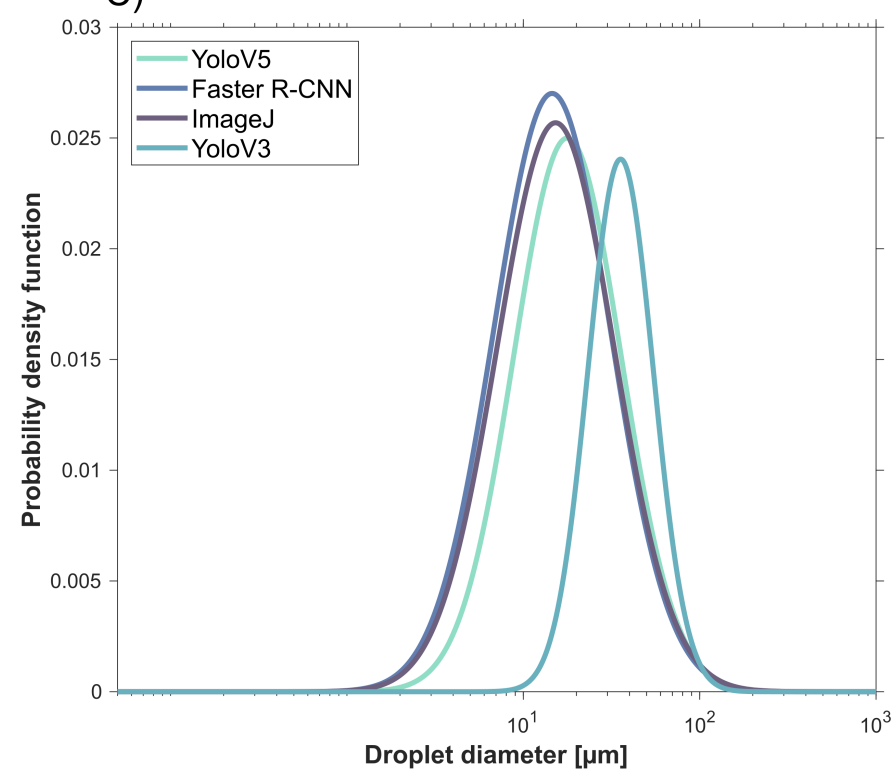

D)

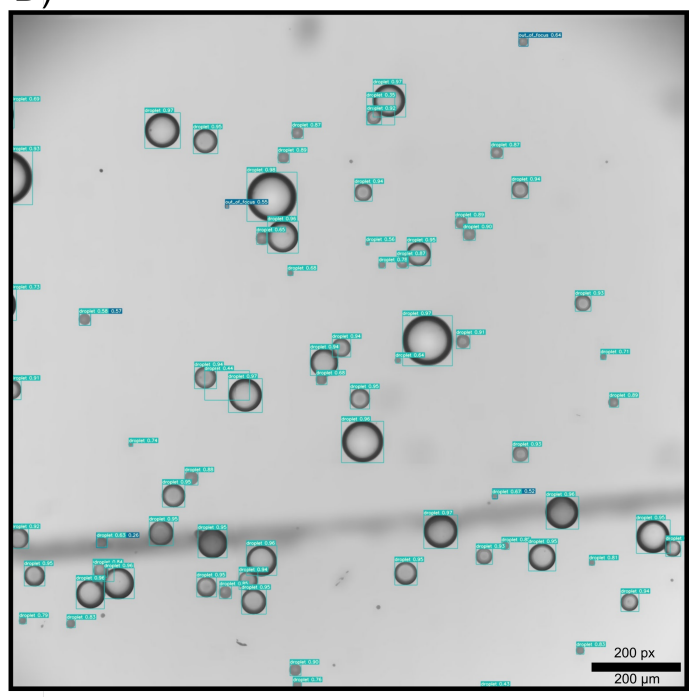

Figure 7: Droplet size distributions shown by a probability density function for A) fine emulsion with B) YOLOv5 inference and C) a coarse emulsion with D) YOLOv5 inference. Inference results from ImageJ, YOLOv3 and Faster $\mathrm{R}-\mathrm{CNN}$ are available in Figure $\mathrm{S} 7$. 
droplets between 1 and 5 pixels, especially so in agglomerated groups, and thus it follows that model architecture must be addressed. Specifically, if there was some methodological unification between the discretized grid used by YOLOv5 and the sliding window of Faster R-CNN, higher resolution images can be fed to this hybrid architecture which moves across bigger images proposing detections within discretized grids. A non-architectural overhaul would simply involve experimental modification where higher microscope magnification is used with more sensitive stage movements, specifically for micrographs or porous media where precision loss is more prevalent in polydisperse systems, discussed below.

\subsection{Porous Media}

Certain emulsion science phenomena often extend to understanding system dynamics outside of various flow regimes, such as in model systems with implications for extraction or retention characteristics [77]. Microfludics can be an alternative approach to conventional flooding techniques for the studies concerning the transport of emulsions in porous media. The microfluidic studies allow pore-scale observations of droplet transport, whose outcomes could build a fundamental knowledge about droplet retention. However, such microfluidic experiments pose a challenge when it comes to image analysis as the microfluidic chips representing porous media have a complex geometry. We employ porous media studies in tandem with the integration of these CNNs into our image analysis pipeline and thus the following is newly reported data.

In order to simulate porous structures, the microfluidic chip architecture is modified into a topographical network of etched features intended to impede droplet transport, shown in Figure 2B. The addition of the rock-like feature matrix raises the degree of complexity for traditional image analysis techniques. The complexity arises from the pixel intensities present at the edges of the etch features which are also highly similar to pixel ensembles present at droplets interfaces. The circular Hough transform can still be applied for droplet detection with a fair level of robustness, despite the presence of the porous matrix, albeit with stringent limitations. Firstly, as in the prior Hough transform implementations in our various microfluidic systems, the method struggles with detection of objects $<5$ pixels. Additionally, the etched features of the chip exhibited enough circularity to become challenging for the Hough transform to distinguish between them and droplets, and required sacrificing data integrity to filter them from the droplet statistics. The data processing is time and resource intensive, as typically processing time was $2.5-3$ seconds per image, for a dataset of 25000 images from various porous media experiments. ImageJ's "Analyze Particles" suite suffered from the same limitations as the Hough transform in these experiments and produced less quality data than the latter.

Despite the elevated level of background complexity present in these microfluidic systems, we posit the CNNs from the prior detection experiments can also be implemented as a competitive or superior method for droplet detection and data harvesting. Initially, in our prototype training and validation set, the models were not exposed to data from these porous media experiments. The best inference results had a broad mix of accurate detections of droplets and false detections of chip features. Similarly to the implementation of the Hough transform, where prominently convex chip features must be specifically filtered out, the detection of these edges must be accounted for by training out this type of false inference. The weights produced by our final training and validation dataset, shown in Figure 2, droplets from various annotated porous media experiments were the most representative type of droplet in the entire dataset. The nature of these experiments includes inhibition of droplet transport and thus the concentration of droplets at the face of the network is usually high compared to flow cell microfluidics, thus increasing the detection task complexity even further. Inference was performed on monodisperse droplets in porous media with all three CNNs, and the results are shown in Figure 8. Absolute droplet counts can be seen in Table 6 for monodisperse and polydisperse droplets retained in the porous media chip, obtained for each detection method.

Table 6: The number of detected droplets in mono and polydisperse in porous media using various methods.

\begin{tabular}{cccccc}
\hline Emulsion Homogeneity & Hough & ImageJ & Faster R-CNN & YOLOv3 & YOLOv5 \\
\hline Monodisperse & 2989 & 2765 & 2658 & 2857 & 2844 \\
Polydisperse & 15762 & 14349 & 16783 & 15233 & 16358 \\
\hline
\end{tabular}

The glaring statistical difference between the reported monodisperse distributions is the appearance of two major diameter peaks. YOLOv3 and ImageJ both report that the majority of detected droplets have diameter centered at approximately $42.5 \mu \mathrm{m}$. The Hough transform shares slight overlap between these with the most Gaussian-like distribution which shared overlap between both mean peaks. Contrastingly, both Faster R-CNN and YOLOv5 mainly reported a bulk of the diameters approximately between 47 and $50 \mu \mathrm{m}$. The peak shift is explained by the placement of the detection or bounding box around or within the droplet. The Hough transform, ImageJ and YOLOv3 detect droplets by bounding within the droplet interface. The former two categorically eliminate the interface and thus 

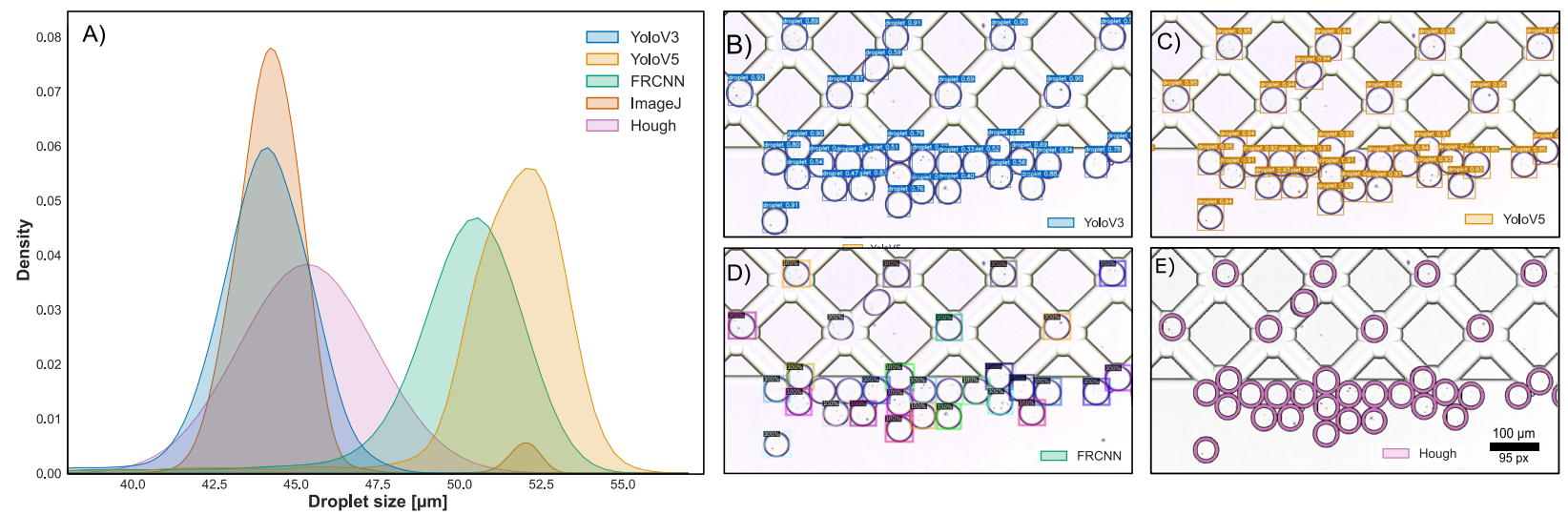

Figure 8: A) Shows kernel density estimation plot for all detection methods, including the traditional approaches, for a monodisperse emulsion injected into a porous media matrix. Inference results performed by by B) YOLOv3, C) YOLOv5, D) Faster R-CNN, E) Hough Transform.

shave several microns from a true estimation of the entire droplet. Faster R-CNN and YOLOv5 bound the droplet directly at the interface thus preserving the true diameter measurement without any further assumptive post-processing. Among these methods, despite the bounding precision of Faster R-CNN, it fails to detect significantly more droplets than all the other methods. We consider the Hough transform the reference method for droplet count or something very near "ground-truth" as we manually checked this entire dataset for missed detections. For this monodisperse set of droplets retained in the porous matrix, the Hough transform detected 2989 droplets. In terms of raw counts, YOLOv3 and YOLOv5 both outperform the entire ImageJ pipeline, which includes the "Analyze Particles" function and data post-processing. Generally, the Hough transform exists in the domain of accurate number of raw detections but algorithmically it performs detection of droplets within the interface, thereby shifting the true size distribution by approximately $8 \mu \mathrm{m}$, which is a drawback in ImageJ and YOLOv3 as well. Training YOLOv3 further will most likely result in some degree of overfitting for future predictions as precision and recall already converge well with loss over time, with the model reporting near 100\% confidence for detected droplets. The overfitting risk exists within Faster R-CNN as well due to confidence levels of $100 \%$ despite a significant amount of missed detections. A solution could be to create a dataset specifically for porous media systems or other more complex microfluidic architectures that naturally augment the detection task with confusing edge morphologies that present droplet-like interfaces. However, YOLOv3 exhibits divergent behavior in the secondary porous media experiment, this time using a polydisperse emulsion. The results of this polydisperse retention experiment are shown in Figure 9.

The polydispersity of the tested systems leads to a wide range of droplet diameters, and we thus use pixel values instead to emphasize the limitations of each detection method. Similarly to the monodisperse porous media results, we can use the Hough transform as a template for something near true droplet count in the system. Trend-wise even as the droplet size distribution broadens, there is distribution symmetry between YOLOv3 and ImageJ. The same methodological issue exists where for the Hough transform, ImageJ and YOLOv3 the droplets are mainly detected within their interfaces which again contributes to an underestimation of the true diameter on the order of $8 \mu \mathrm{m}$, approximately. It follows, that Faster R-CNN and YOLOv5 still generally infer the bounding box around the droplet interface. The level of polydispersity in this emulsion is clear due to multiple mean peaks which develop alongside bigger droplets beyond 50 $\mu \mathrm{m}$ diameter, which are mainly detected by Faster R-CNN and YOLOv5. YOLOv3 has the least "tail-like" behavior because it has the least consistent internal ruleset regarding bounding boxes as a function of droplet size. While it detects within the interface for larger droplets, as the diameter falls below $30 \mu \mathrm{m}$, YOLOv3 begins to overestimate the size of the droplet and fails at detecting small objects, as in the other experiments. Contrastingly, Faster R-CNN significantly overfits detections for smaller objects and often falsely detects either contaminants or small chip features with localized lighting artifacts as droplets contributing to a highly overestimated true droplet count. Most of this is observable in the tail under $10 \mu \mathrm{m}$. YOLOv5 also occasionally falsely reports artifacts or contaminants as small objects albeit with very low confidence compared to true small droplets with high confidence; therefore making it entirely feasible to filter false detections quickly. Even though we consider the Hough transform a relative baseline, it struggles with very small objects in this size range and we estimate that in more polydisperse droplet sets YOLOv5 is actually 5\% more accurate at reporting true droplet counts due to the surprising detection aptitude across the entire size spectrum. ImageJ also struggles with small droplets for several reasons. Firstly, because the chip features for this microfluidic system must not be detected, ImageJ requires thresholding to do this and in the same pre-processing step, the smaller 
A PREPRINT - JUNE 1, 2021

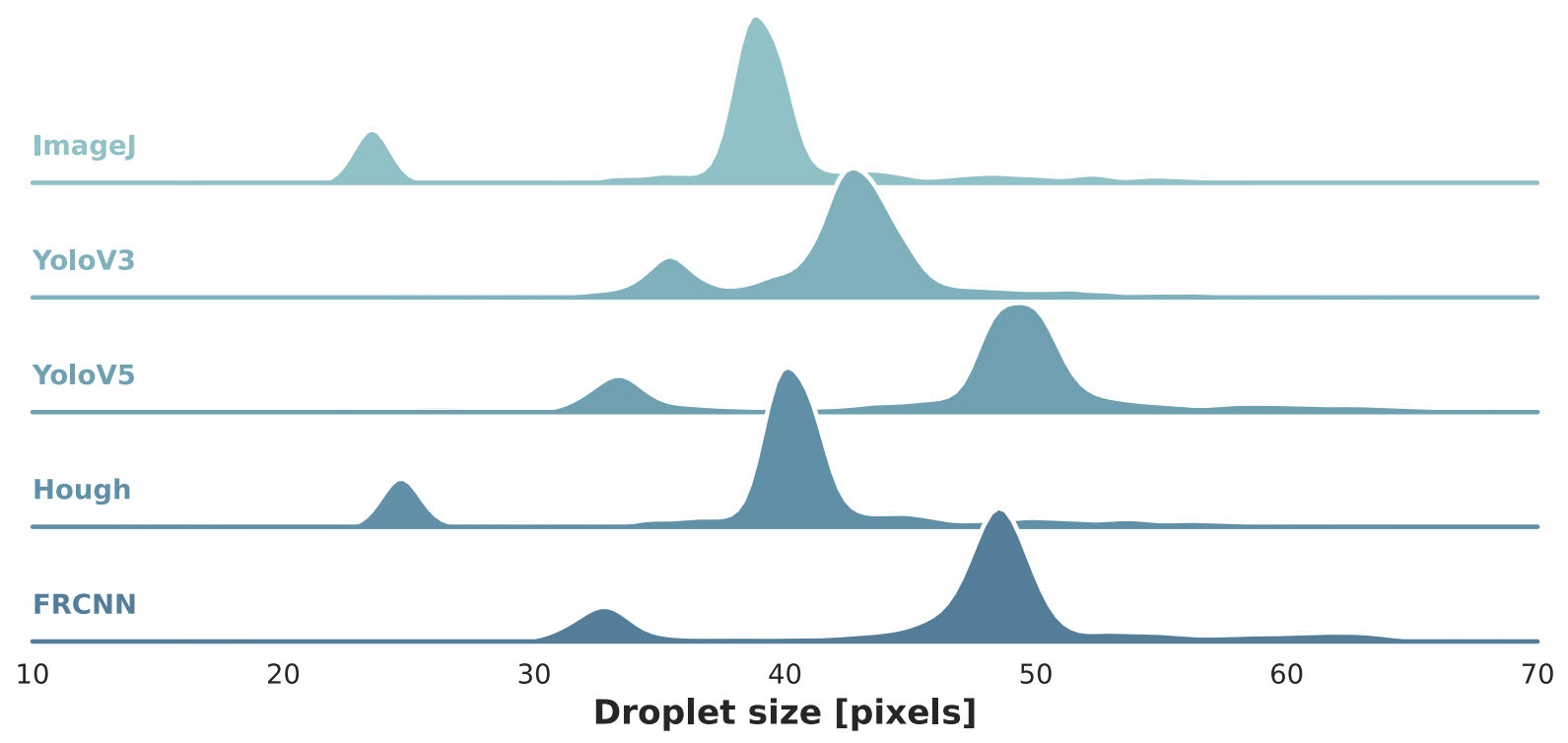

Figure 9: Size distributions for data gathered from a porous media experiment which was injected with a polydisperse emulsion.

droplets are immediately removed from consideration. Additionally, the inherent convexity of the void space between circular droplets at agglomerated clusters is always detected and is also filtered.

All methods falter when droplets appear in a microfluidic chip with features that are highly visually representative of droplet interfaces. Despite this, all methods generally report distribution symmetry, and being able to perform detection either inside or at the droplet interface is the main factor which affects the variance between the detections. Ultimately, both YOLOv3 and v5 can perform per-frame inference in less than $0.02 \mathrm{~s}$ which unlocks access to the same statistics as classical methods in fractions of the time required by the latter.

\section{Conclusions}

Leveraging the computational advantage of various deep-learning approaches and CNN architectures in analysis pipelines is clearly evolving to be an intensely focused area of research. Throughout this work, we have shown that the three CNN's, Faster R-CNN, YOLOv3 and YOLOv5, can be trained on comprehensive microfluidic droplet datasets and then deployed as droplet detectors in a gamut of microfluidic systems and experiments. The models all show viability throughout the systems we tested, in comparison to data acquired via traditional methods and software packages (i.e. Hough Transform implemented in Matlab and the Particle Analyzer suite in ImageJ). Overall, YOLOv5 appears more robust compared to other two models, as it almost always provided precise bounding boxes in comparison to the traditional methods and outperformed these approaches as the detection task became more complex, as in the porous media systems. Faster R-CNN rivaled YOLOv5 in many of the experiments but sometimes overfit and falsely detected or completely missed obvious detections while also underperformed compared to YOLOv5's blazingly quick $10 \mathrm{~ms}$ per frame inference time. The former is still valuable as segmentation can be integrated via Mask R-CNN, and could potentially open the door for more complex levels of analysis in 3D droplet systems where the z-axis contributes to droplet agglomeration and occlusion. YOLOv5's consistent outperformance maintains statistical fidelity throughout the analysis pipeline at a fraction of the time investment and thus this method has become our primary droplet detection tool for many future microfluidic experiments. While the smallest version of YOLOv5 offers inference capable of live object detection at moderate framerates on high speed cameras, the model can be further condensed due to the fundamental nature of this well-established computer vision task. Decreasing the number of parameters will reduce the computational cost of pushing data through the model, thus decreasing its GPU memory footprint while still maintaining detection robustness in recording regimes greater than $200 \mathrm{fps}$. 


\section{Acknowledgements and Contributions}

\subsection{Acknowledgements}

The Research Council of Norway is acknowledged for the support to the Norwegian Micro- and Nano-Fabrication Facility, NorFab, project number 295864.

The authors would also like to acknowledge Gisle Øye for supporting the work and reviewing this manuscript.

\subsection{Author Contributions}

Conceptualization: GR, IA. Theoretical and Experimental discussion: GR, IA, EU, MD, BAG. Experimentation and data collection: GR, IA, MD. Data curation: GR, IA, MD. Traditional image analysis (ImageJ/MATLAB): IA, MD. Software and script development for models: GR, EU. Formal data analysis: GR, IA, MD. Writing - original draft: GR, IA, MD, EU. Critical revision and editing: GR, IA, MD, BAG. Supervision: BAG.

\subsection{Data Availability}

The data used for this work including annotations for the droplet and coalescence training and validation sets is avaialable upon request. The code used to train and analyze inference results is also available upon request.

\subsection{Funding}

The authors declare no competing financial interest.

\section{References}

[1] Paul C. Hiemenz and Raj Rajagopalan. Principles of Colloid and Surface Chemistry. CRC Press, Boca Raton, 1997.

[2] Shelley Lynn Anna. Droplets and bubbles in microfluidic devices. Annual Review of Fluid Mechanics, 48(1):285309, 2016.

[3] Nicolas Bremond and Jérôme Bibette. Exploring emulsion science with microfluidics. Soft Matter, 8:10549-10559, 2012.

[4] Luoran Shang, Yao Cheng, and Yuanjin Zhao. Emerging droplet microfluidics. Chemical Reviews, 117(12):79648040, 2017. PMID: 28537383.

[5] Michel Versluis. High-speed imaging in fluids. Experiments in Fluids, 54:1458, 2013.

[6] Koen van Dijke, Riëlle de Ruiter, Karin Schroën, and Remko Boom. The mechanism of droplet formation in microfluidic edge systems. Soft Matter, 6:321-330, 2010.

[7] Tomasz Glawdel, Caglar Elbuken, and Carolyn L. Ren. Droplet formation in microfluidic t-junction generators operating in the transitional regime. i. experimental observations. Phys. Rev. E, 85:016322, Jan 2012.

[8] Evelien W. M. Kemna, Rogier M. Schoeman, Floor Wolbers, Istvan Vermes, David A. Weitz, and Albert van den Berg. High-yield cell ordering and deterministic cell-in-droplet encapsulation using dean flow in a curved microchannel. Lab Chip, 12:2881-2887, 2012.

[9] Tian Wang, Simon Ivar Andersen, and Alexander Shapiro. Coalescence of oil droplets in microchannels under brine flow. Colloids and Surfaces A: Physicochemical and Engineering Aspects, 598:124864, 2020.

[10] HK Yuen, John Princen, John Illingworth, and Josef Kittler. Comparative study of hough transform methods for circle finding. Image and vision computing, 8(1):71-77, 1990.

[11] John Illingworth and Josef Kittler. The adaptive hough transform. IEEE Transactions on Pattern Analysis and Machine Intelligence, (5):690-698, 1987.

[12] Mohamed Rizon, Yazid Haniza, Saad Puteh, Ali Yeon, Md Shakaff, Saad Abdul Rahman, Manasori Sugisaka, Yaacob Sazali, Mamat M Rozailan, and M Karthigayan. Object detection using circular hough transform. 2005.

[13] Miné Zantow, Ronald Dendere, and Tania S Douglas. Image-based analysis of droplets in microfluidics. In 2013 35th Annual International Conference of the IEEE Engineering in Medicine and Biology Society (EMBC), pages 1776-1779. IEEE, 2013. 
[14] Philippe QN Vo, Mathieu C Husser, Fatemeh Ahmadi, Hugo Sinha, and Steve CC Shih. Image-based feedback and analysis system for digital microfluidics. Lab on a Chip, 17(20):3437-3446, 2017.

[15] Massimo Minervini, Hanno Scharr, and Sotirios A Tsaftaris. Image analysis: the new bottleneck in plant phenotyping [applications corner]. IEEE signal processing magazine, 32(4):126-131, 2015.

[16] Ian Goodfellow, Yoshua Bengio, Aaron Courville, and Yoshua Bengio. Deep learning, volume 1. MIT press Cambridge, 2016.

[17] Yann LeCun, Yoshua Bengio, and Geoffrey Hinton. Deep learning. nature, 521(7553):436-444, 2015.

[18] David G Lowe. Distinctive image features from scale-invariant keypoints. International journal of computer vision, 60(2):91-110, 2004.

[19] Navneet Dalal and Bill Triggs. Histograms of oriented gradients for human detection. In 2005 IEEE computer society conference on computer vision and pattern recognition (CVPR'05), volume 1, pages 886-893. Ieee, 2005.

[20] Rainer Lienhart and Jochen Maydt. An extended set of haar-like features for rapid object detection. In Proceedings. international conference on image processing, volume 1, pages I-I. IEEE, 2002.

[21] David A. Van Valen, Takamasa Kudo, Keara M. Lane, Derek N. Macklin, Nicolas T. Quach, Mialy M. DeFelice, Inbal Maayan, Yu Tanouchi, Euan A. Ashley, and Markus W. Covert. Deep learning automates the quantitative analysis of individual cells in live-cell imaging experiments. PLOS Computational Biology, 12(11):1-24, 112016.

[22] Stuart Berg, Dominik Kutra, Thorben Kroeger, Christoph N. Straehle, Bernhard X. Kausler, Carsten Haubold, Martin Schiegg, Janez Ales, Thorsten Beier, Markus Rudy, Kemal Eren, Jaime I. Cervantes, Buote Xu, Fynn Beuttenmueller, Adrian Wolny, Chong Zhang, Ullrich Koethe, Fred A. Hamprecht, and Anna Kreshuk. ilastik: interactive machine learning for (bio)image analysis. Nature Methods, 16(12):1226-1232, Dec 2019.

[23] D Waithe, JM Brown, K Reglinski, I Diez-Sevilla, D Roberts, and Christian Eggeling. Object detection networks and augmented reality for cellular detection in fluorescence microscopy acquisition and analysis. bioRxiv, 2019.

[24] Ilida Suleymanova, Tamas Balassa, Sushil Tripathi, Csaba Molnar, Mart Saarma, Yulia Sidorova, and Peter Horvath. A deep convolutional neural network approach for astrocyte detection. Scientific Reports, 8:1278, 2018. DOI : $10.1038 / \mathrm{s} 41598-018-31284-\mathrm{x}$.

[25] Claire McQuin, Allen Goodman, Vasiliy Chernyshev, Lee Kamentsky, Beth A. Cimini, Kyle W. Karhohs, Minh Doan, Liya Ding, Susanne M. Rafelski, Derek Thirstrup, Winfried Wiegraebe, Shantanu Singh, Tim Becker, Juan C. Caicedo, and Anne E. Carpenter. Cellprofiler 3.0: Next-generation image processing for biology. PLOS Biology, 16(7):1-17, 072018.

[26] Ahmad Paintdakhi, Bradley Parry, Manuel Campos, Irnov Irnov, Johan Elf, Ivan Surovtsev, and Christine JacobsWagner. Oufti: an integrated software package for high-accuracy, high-throughput quantitative microscopy analysis. Molecular Microbiology, 99(4):767-777, 2016.

[27] Imanol Luengo, Michele C. Darrow, Matthew C. Spink, Ying Sun, Wei Dai, Cynthia Y. He, Wah Chiu, Tony Pridmore, Alun W. Ashton, Elizabeth M.H. Duke, Mark Basham, and Andrew P. French. Survos: Super-region volume segmentation workbench. Journal of Structural Biology, 198(1):43-53, 2017.

[28] Michael Held, Michael H. A. Schmitz, Bernd Fischer, Thomas Walter, Beate Neumann, Michael H. Olma, Matthias Peter, Jan Ellenberg, and Daniel W. Gerlich. Cellcognition: time-resolved phenotype annotation in high-throughput live cell imaging. Nature Methods, 7(9):747-754, Sep 2010.

[29] Ilya Belevich, Merja Joensuu, Darshan Kumar, Helena Vihinen, and Eija Jokitalo. Microscopy image browser: A platform for segmentation and analysis of multidimensional datasets. PLOS Biology, 14(1):1-13, 012016.

[30] Raphaël Marée, Loïc Rollus, Benjamin Stévens, Renaud Hoyoux, Gilles Louppe, Rémy Vandaele, Jean-Michel Begon, Philipp Kainz, Pierre Geurts, and Louis Wehenkel. Collaborative analysis of multi-gigapixel imaging data using cytomine. Bioinformatics (Oxford, England), 32(9):1395-1401, May 2016. 26755625[pmid].

[31] Y.J. Heo, D. Lee, J. Kang, and W.K. Chung. Real-time Image Processing for Microscopy-based Label-free Imaging Flow Cytometry in a Microfluidic Chip. Scientific Reports, 7:790-784, 2017. https://doi .org/10. 1038/s41598-017-11534-0.

[32] P. Hadikhani, S.M. Borhani, N.and H. Hashemi, and D. Psaltis. Learning from droplet flows in microfluidic channels using deep neural networks. Scientific Reports, 9:1-7, 2019. https://doi.org/10.1038/ s41598-019-44556-x.

[33] Ross Girshick, Jeff Donahue, Trevor Darrell, and Jitendra Malik. Rich feature hierarchies for accurate object detection and semantic segmentation. In Proceedings of the IEEE conference on computer vision and pattern recognition, pages 580-587, 2014. 
[34] Zhong-Qiu Zhao, Peng Zheng, Shou-tao Xu, and Xindong Wu. Object detection with deep learning: A review. IEEE transactions on neural networks and learning systems, 30(11):3212-3232, 2019.

[35] Alex Krizhevsky, Ilya Sutskever, and Geoffrey E Hinton. Imagenet classification with deep convolutional neural networks. Advances in neural information processing systems, 25:1097-1105, 2012.

[36] Robert Hecht-Nielsen. Theory of the backpropagation neural network. In Neural networks for perception, pages 65-93. Elsevier, 1992.

[37] Xu Sun, Xuancheng Ren, Shuming Ma, and Houfeng Wang. meprop: Sparsified back propagation for accelerated deep learning with reduced overfitting. In International Conference on Machine Learning, pages 3299-3308. PMLR, 2017.

[38] Riccardo Porotti, Dario Tamascelli, Marcello Restelli, and Enrico Prati. Coherent transport of quantum states by deep reinforcement learning. Communications Physics, 2(1):1-9, 2019.

[39] Hao Li, Zheng Xu, Gavin Taylor, Christoph Studer, and Tom Goldstein. Visualizing the loss landscape of neural nets. arXiv preprint arXiv:1712.09913, 2017.

[40] Tom Dietterich. Overfitting and undercomputing in machine learning. ACM computing surveys (CSUR), 27(3):326327, 1995.

[41] Nitish Srivastava, Geoffrey Hinton, Alex Krizhevsky, Ilya Sutskever, and Ruslan Salakhutdinov. Dropout: a simple way to prevent neural networks from overfitting. The journal of machine learning research, 15(1):1929-1958, 2014.

[42] Shaoqing Ren, Kaiming He, Ross Girshick, and Jian Sun. Faster r-cnn: Towards real-time object detection with region proposal networks. arXiv preprint arXiv:1506.01497, 2015.

[43] Joseph Redmon, Santosh Divvala, Ross Girshick, and Ali Farhadi. You only look once: Unified, real-time object detection. In Proceedings of the IEEE conference on computer vision and pattern recognition, pages 779-788, 2016.

[44] Ross Girshick. Fast r-cnn. In Proceedings of the IEEE international conference on computer vision, pages 1440-1448, 2015.

[45] Christian Szegedy, Alexander Toshev, and Dumitru Erhan. Deep neural networks for object detection. 2013.

[46] Pedro O Pinheiro, Ronan Collobert, and Piotr Dollár. Learning to segment object candidates. arXiv preprint arXiv:1506.06204, 2015.

[47] Christian Szegedy, Scott Reed, Dumitru Erhan, Dragomir Anguelov, and Sergey Ioffe. Scalable, high-quality object detection. arXiv preprint arXiv:1412.1441, 2014.

[48] Dumitru Erhan, Christian Szegedy, Alexander Toshev, and Dragomir Anguelov. Scalable object detection using deep neural networks. In Proceedings of the IEEE conference on computer vision and pattern recognition, pages 2147-2154, 2014.

[49] Donggeun Yoo, Sunggyun Park, Joon-Young Lee, Anthony S Paek, and In So Kweon. Attentionnet: Aggregating weak directions for accurate object detection. In Proceedings of the IEEE International Conference on Computer Vision, pages 2659-2667, 2015.

[50] Mahyar Najibi, Mohammad Rastegari, and Larry S Davis. G-cnn: an iterative grid based object detector. In Proceedings of the IEEE conference on computer vision and pattern recognition, pages 2369-2377, 2016.

[51] Marcin Dudek, Are Bertheussen, Thomas Dumaire, and Gisle Øye. Microfluidic tools for studying coalescence of crude oil droplets in produced water. Chemical Engineering Science, 191:448-458, 2018.

[52] Marcin Dudek, Julien Chicault, and Gisle Øye. Microfluidic investigation of crude oil droplet coalescence: Effect of oil/water composition and droplet aging. Energy \& Fuels, 34(5):5110-5120, 2020.

[53] Marcin Dudek, Diana Fernandes, Eirik Helno Herø, and Gisle Øye. Microfluidic method for determining dropdrop coalescence and contact times in flow. Colloids and Surfaces A: Physicochemical and Engineering Aspects, 586:124265, 2020.

[54] Marcin Dudek, Kelly Muijlwijk, Karin Schroën, and Gisle Øye. The effect of dissolved gas on coalescence of oil drops studied with microfluidics. Journal of Colloid and Interface Science, 528:166-173, 2018.

[55] Antoni Buades, Bartomeu Coll, and Jean-Michel Morel. Non-Local Means Denoising. Image Processing On Line, 1:208-212, 2011. https://doi.org/10.5201/ipol.2011.bcm_nlm.

[56] Yuxin Wu, Alexander Kirillov, Francisco Massa, Wan-Yen Lo, and Ross Girshick. Detectron2. https ://github . com/facebookresearch/detectron2, 2019. 
[57] Glenn Jocher. ultralytics/yolov5: v3.1 - Bug Fixes and Performance Improvements. https://github.com/ ultralytics/yolov5, October 2020.

[58] Tsung-Yi Lin, Michael Maire, Serge Belongie, James Hays, Pietro Perona, Deva Ramanan, Piotr Dollár, and C Lawrence Zitnick. Microsoft coco: Common objects in context. In European conference on computer vision, pages 740-755. Springer, 2014.

[59] Piotr Garstecki, Michael J. Fuerstman, Howard A. Stone, and George M. Whitesides. Formation of droplets and bubbles in a microfluidic t-junction—scaling and mechanism of break-up. Lab Chip, 6:437-446, 2006.

[60] Joshua D. Tice, Adam D. Lyon, and Rustem F. Ismagilov. Effects of viscosity on droplet formation and mixing in microfluidic channels. Analytica Chimica Acta, 507(1):73-77, 2004. Microfluidics and Lab - On - a - Chip.

[61] Joeska Husny and Justin J. Cooper-White. The effect of elasticity on drop creation in t-shaped microchannels. Journal of Non-Newtonian Fluid Mechanics, 137(1):121-136, 2006. Extensional Flow.

[62] Taotao Fu, Yining Wu, Youguang Ma, and Huai Z. Li. Droplet formation and breakup dynamics in microfluidic flow-focusing devices: From dripping to jetting. Chemical Engineering Science, 84:207-217, 2012.

[63] Lucas Frenz, Abdeslam ElHarrak, Matthias Pauly, Sylvie Bégin-Colin, AndrewD. Griffiths, and Jean-Christophe Baret. Droplet-based microreactors for the synthesis of magnetic iron oxide nanoparticles. Angewandte Chemie International Edition, 47(36), 2008.

[64] Koen C van Dijke, Gert Veldhuis, Karin Schroën, and Remko M Boom. Simultaneous formation of many droplets in a single microfluidic droplet formation unit. AIChE journal, 56(3):833-836, 2010.

[65] Marcin Dudek, Hanne Skudal Ullaland, Amandine Wehrle, and Gisle Øye. Microfluidic testing of flocculants for produced water treatment: Comparison with other methodologies. Water Research X, 9:100073, 2020.

[66] Thomas Krebs, Karin Schroën, and Remko Boom. Coalescence dynamics of surfactant-stabilized emulsions studied with microfluidics. Soft Matter, 8:10650-10657, 2012.

[67] Kelly Muijlwijk, Ivanna Colijn, Herditya Harsono, Thomas Krebs, Claire Berton-Carabin, and Karin Schroën. Coalescence of protein-stabilised emulsions studied with microfluidics. Food Hydrocolloids, 70:96-104, 2017.

[68] Jean-Christophe Baret, Felix Kleinschmidt, Abdeslam El Harrak, and Andrew D. Griffiths. Kinetic aspects of emulsion stabilization by surfactants: A microfluidic analysis. Langmuir, 25(11):6088-6093, 2009.

[69] Taotao Fu, Youguang Ma, and Huai Z. Li. Bubble coalescence in non-newtonian fluids in a microfluidic expansion device. Chemical Engineering and Processing: Process Intensification, 97:38-44, 2015.

[70] Anja Schröder, Joris Sprakel, Karin Schroën, Joep N. Spaen, and Claire C. Berton-Carabin. Coalescence stability of pickering emulsions produced with lipid particles: A microfluidic study. Journal of Food Engineering, 234:63-72, 2018.

[71] Thomas Krebs, Karin Schroen, and Remko Boom. A microfluidic method to study demulsification kinetics. Lab Chip, 12:1060-1070, 2012.

[72] Bee Lim, Sanghyun Son, Heewon Kim, Seungjun Nah, and Kyoung Mu Lee. Enhanced deep residual networks for single image super-resolution. In Proceedings of the IEEE conference on computer vision and pattern recognition workshops, pages 136-144, 2017.

[73] Qianqian Zhou, Yue Sun, Shiting Yi, Kai Wang, and Guangsheng Luo. Investigation of droplet coalescence in nanoparticle suspensions by a microfluidic collision experiment. Soft Matter, 12:1674-1682, 2016.

[74] Jianlong Wang, Say Hwa Tan, Anh V. Nguyen, Geoffrey M. Evans, and Nam-Trung Nguyen. A microfluidic method for investigating ion-specific bubble coalescence in salt solutions. Langmuir, 32(44):11520-11524, 2016.

[75] Jianlong Wang, Adrian J. T. Teo, Say H. Tan, Geoffrey M. Evans, Nam-Trung Nguyen, and Anh V. Nguyen. Influence of interfacial gas enrichment on controlled coalescence of oil droplets in water in microfluidics. Langmuir, 35(10):3615-3623, 2019.

[76] Yin-Ting $\mathrm{Hu}$, Yuwen Ting, Jing-Yu Hu, and Shu-Chen Hsieh. Techniques and methods to study functional characteristics of emulsion systems. Journal of Food and Drug Analysis, 25(1):16-26, 2017. Dietary Natural Compounds.

[77] Q. Liu, B. Zhao, and J. C. Santamarina. Particle migration and clogging in porous media: A convergent flow microfluidics study. Journal of Geophysical Research: Solid Earth, 124(9):9495-9504, 2019. 